\title{
Catalytic Enantioselective Synthesis of Quaternary Stereocenters via Intermolecular C-Acylation of Silyl Ketene Acetals: Dual Activation of the Electrophile and the Nucleophile
}

\author{
Ara H. Mermerian and Gregory C. Fu* \\ Department of Chemistry, Massachusetts Institute of Technology, \\ Cambridge, Massachusetts 02139
}

\section{Supporting Information}

\section{General}

THF, $\mathrm{CH}_{2} \mathrm{Cl}_{2}$, and $\mathrm{Et}_{2} \mathrm{O}$ were purified by passage through a neutral alumina column. $\mathrm{Ac}_{2} \mathrm{O}$ was distilled from phosphorus pentoxide. Benzoic anhydride was recrystallized from $\mathrm{Et}_{2} \mathrm{O}$. $\left[\mathrm{Me}_{4} \mathrm{~N}\right] \mathrm{OAc}$ (Alfa Aesar) was purified by recrystallization from 2:1 $\mathrm{CH}_{3} \mathrm{CN} / \mathrm{CH}_{2} \mathrm{Cl}_{2} \cdot n$-BuLi (Alfa Aesar) was titrated with diphenylacetic acid (Aldrich) prior to each use. Methylene chloride- $d_{2}$ (Cambridge Isotope Laboratories) was distilled from $\mathrm{CaH}_{2}$. Phenylacetic acid (Aldrich), 4-methoxyphenylacetic acid (Avocado), 4-trifluoromethylphenylacetic acid (Avocado), o-tolylacetic acid (Avocado), naphthalene-1-acetic acid (Avocado), thiophene-2-acetic acid (Avocado), thiophene-3acetic acid (Avocado), 1-methyl-3-indoleacetic acid (Aldrich), 1,3,2-dioxathiolane 2,2dioxide (Aldrich), diisopropylamine (Aldrich), 1,4-dioxane (Aldrich), $\mathrm{Me}_{3} \mathrm{SiCl}$ (Avocado), isobutylene oxide (TCI), chloroform (Mallinckrodt), pentane (Burdick \& Jackson), hydrochloric acid (Fisher), bromine (Fluka), $\mathrm{FeBr}_{3}$ (Strem), AgSbF 6 (Strem), oxalyl chloride (Aldrich), 2-methyl-2-propanol (Aldrich), $\mathrm{NEt}_{3}$ (EM Science), N,Ndimethylformamide (Aldrich), 2-phenylbutyric acid (Aldrich), tetrabutylammonium 
chloride (TCI), acetonitrile (Aldrich), and absolute EtOH (Pharmco) were used as received. Catalysts 1-4 were prepared as previously reported. ${ }^{1}$

All experiments were conducted under an argon or nitrogen atmosphere in ovendried glassware with magnetic stirring, unless otherwise specified.

(1) (a) Ruble, J. C.; Fu, G. C. J. Org. Chem. 1996, 61, 7230-7231. (b) Ruble, J. C.; Latham, H. A.; Fu, G. C. J. Am. Chem. Soc. 1997, 119, 1492-1493. (c) Ruble, J. C.; Fu, G. C. J. Am. Chem. Soc. 1998, 120, 11532-11533. (d) Tao, B.; Ruble, J. C.; Hoic, D. A.; Fu, G. C. J. Am. Chem. Soc. 1999, 121, 5091-5092. 


\section{Preparation of Esters}

These reactions have not been optimized.

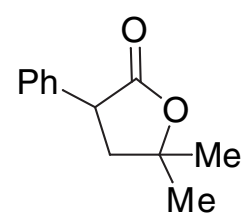

A solution of $n$-BuLi (in hexanes; $75.6 \mathrm{~mL}, 185 \mathrm{mmol}$ ) was added via syringe to a -78 ${ }^{\circ} \mathrm{C}$ solution of diisopropylamine $(25.9 \mathrm{~mL}, 185 \mathrm{mmol})$ in THF $(100 \mathrm{~mL})$. This solution was stirred at $-78^{\circ} \mathrm{C}$ for 45 minutes, and then a solution of phenylacetic acid (12.0 g, $88.1 \mathrm{mmol})$ in THF $(75 \mathrm{~mL})$ was added via cannula. The reaction mixture was stirred at $-78{ }^{\circ} \mathrm{C}$ for 20 minutes, then warmed to room temperature and stirred for 2 hours. The solution was then cooled to $0{ }^{\circ} \mathrm{C}$, and neat isobutylene oxide $(7.90 \mathrm{~mL}, 88.1 \mathrm{mmol})$ was added via syringe, resulting in a clear yellow solution, which was stirred for 12 hours at room temperature. Water $(50 \mathrm{~mL})$ was then added, resulting in a clear colorless solution, which was refluxed for 2 hours. Then, the reaction mixture was cooled to room temperature and washed with $\mathrm{Et}_{2} \mathrm{O}(3 \times 150 \mathrm{~mL})$. The aqueous layer was diluted with 95\% EtOH (100 mL), acidified with concentrated $\mathrm{HCl}(25 \mathrm{~mL})$, and refluxed for 3 hours. Then, it was cooled to room temperature and extracted with $\mathrm{CHCl}_{3}(3 \times 200$ $\mathrm{mL})$. The $\mathrm{CHCl}_{3}$ layer was washed with saturated aqueous $\mathrm{NaHCO}_{3}(2 \times 100 \mathrm{~mL})$, then dried $\left(\mathrm{Na}_{2} \mathrm{SO}_{4}\right)$ and concentrated. The resulting white solid was recrystallized from $\mathrm{Et}_{2} \mathrm{O}$ /pentane (9:1) to afford white crystals, which were collected, washed with pentane, and dried under vacuum $(13.7 \mathrm{~g}, 82 \%)$.

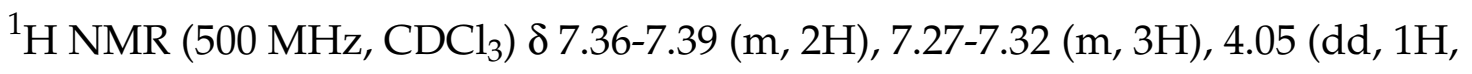
$\mathrm{J}=12.0, \mathrm{~J}=9.5), 2.59$ (dd, 1H, J=12.5, J=9.5), $2.25(\operatorname{app~t}, 1 \mathrm{H}, \mathrm{J}=12.5), 1.56(\mathrm{~s}, 3 \mathrm{H}), 1.51$ (s, $3 \mathrm{H}) .{ }^{13} \mathrm{C}$ NMR $\left(125 \mathrm{MHz}, \mathrm{CDCl}_{3}\right) \delta 176.7,137.2,129.1,128.3,127.7,82.3,47.2,44.4,29.1$, 27.2. FTIR $\left(\mathrm{CH}_{2} \mathrm{Cl}_{2}\right) 3031,2979,2945,1772,1653,1498,1457,1375,1261,1141 \mathrm{~cm}^{-1}$. HRMS (EI, $m / z$ ) calcd. for $\mathrm{C}_{12} \mathrm{H}_{14} \mathrm{O}_{2}\left(\mathrm{M}^{+}\right)$190.0994, found 190.0989. mp 63-66 ${ }^{\circ} \mathrm{C}$. 


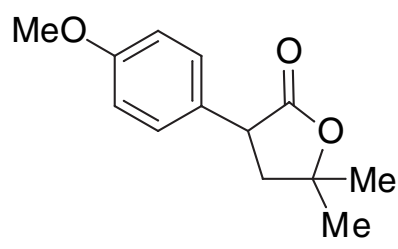

A solution of $n$-BuLi (in hexanes; $48.6 \mathrm{~mL}, 126 \mathrm{mmol}$ ) was added via syringe to a -78 ${ }^{\circ} \mathrm{C}$ solution of diisopropylamine $(17.7 \mathrm{~mL}, 126 \mathrm{mmol})$ in THF $(150 \mathrm{~mL})$. This mixture was stirred at $-78{ }^{\circ} \mathrm{C}$ for 30 minutes, then warmed to $0{ }^{\circ} \mathrm{C}$. A solution of 4 methoxyphenylacetic acid $(7.00 \mathrm{~g}, 42.1 \mathrm{mmol})$ in THF $(50 \mathrm{~mL})$ was added via cannula, and the resulting mixture was warmed to $40{ }^{\circ} \mathrm{C}$ and stirred for 1 hour. Neat isobutylene oxide $(7.90 \mathrm{~mL}, 88.1 \mathrm{mmol})$ was then added via syringe, and the resulting solution was refluxed for 18 hours. The reaction mixture was cooled to $0^{\circ} \mathrm{C}$, and water $(100 \mathrm{~mL})$ was added, resulting in a clear, colorless solution. This mixture was refluxed for 2 hours, then cooled to room temperature, diluted with water $(100 \mathrm{~mL})$, and washed with $\mathrm{Et}_{2} \mathrm{O}(3 \times 150 \mathrm{~mL})$. The aqueous layer was diluted with absolute $\mathrm{EtOH}(100 \mathrm{~mL})$, acidified with $6 \mathrm{~N} \mathrm{HCl}(70 \mathrm{~mL})$, and then refluxed for 4 hours. The mixture was cooled to room temperature and extracted with $\mathrm{CHCl}_{3}(3 \times 200 \mathrm{~mL})$. The $\mathrm{CHCl}_{3}$ layer was washed with saturated aqueous $\mathrm{NaHCO}_{3}(2 \times 100 \mathrm{~mL})$, dried $\left(\mathrm{MgSO}_{4}\right)$, and concentrated. The resulting yellow solid was recrystallized in 3 crops from $\mathrm{Et}_{2} \mathrm{O} / \mathrm{CH}_{2} \mathrm{Cl}_{2} /$ pentane (5:4:50) to afford flocculent white needles, which were collected, washed with pentane, and dried under vacuum $(7.79 \mathrm{~g}, 84 \%)$.

${ }^{1} \mathrm{H}$ NMR (500 MHz, $\left.\mathrm{CDCl}_{3}\right) \delta 7.20(\mathrm{~d}, 2 \mathrm{H}, \mathrm{J}=8.5), 6.90(\mathrm{~d}, 2 \mathrm{H}, \mathrm{J}=9.0), 3.99(\mathrm{dd}, 1 \mathrm{H}$, $\mathrm{J}=12.0, \mathrm{~J}=9.5), 3.80(\mathrm{~s}, 3 \mathrm{H}), 2.55(\mathrm{dd}, 1 \mathrm{H}, \mathrm{J}=12.5, \mathrm{~J}=9.0), 2.20(\mathrm{dd}, 1 \mathrm{H}, \mathrm{J}=12.5, \mathrm{~J}=12.0), 1.54$ $(\mathrm{s}, 3 \mathrm{H}), 1.48(\mathrm{~s}, 3 \mathrm{H}) .{ }^{13} \mathrm{C} \mathrm{NMR}\left(125 \mathrm{MHz}, \mathrm{CDCl}_{3}\right) \delta 177.0,159.1,129.3,129.1,114.5,82.2$, 55.5, 46.4, 44.4, 29.1, 27.1. FTIR $\left(\mathrm{CH}_{2} \mathrm{Cl}_{2}\right)$ 2975, 2935, 2838, 1771, 1653, 1615, 1516, 1375, 1250, $1139 \mathrm{~cm}^{-1}$. HRMS (EI, $\mathrm{m} / \mathrm{z}$ ) calcd. for $\mathrm{C}_{13} \mathrm{H}_{16} \mathrm{O}_{3}\left(\mathrm{M}^{+}\right)$220.1094, found 220.1090. mp $64-68{ }^{\circ} \mathrm{C}$. 


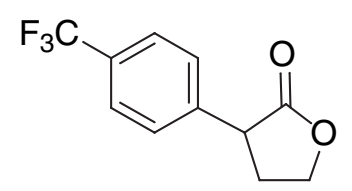

A solution of $n-\mathrm{BuLi}$ (in hexanes; $6.32 \mathrm{~mL}, 17.7 \mathrm{mmol}$ ) was added via syringe to a -78 ${ }^{\circ} \mathrm{C}$ solution of diisopropylamine $(2.48 \mathrm{~mL}, 17.7 \mathrm{mmol})$ in THF $(50 \mathrm{~mL})$. The reaction mixture was stirred at $-78{ }^{\circ} \mathrm{C}$ for 45 minutes, after which a solution of 4trifluoromethylphenylacetic acid $(1.72 \mathrm{~g}, 8.43 \mathrm{mmol})$ in THF $(10 \mathrm{~mL})$ was added via cannula, resulting in a deep-red solution. This mixture was stirred for 45 minutes at -78 ${ }^{\circ} \mathrm{C}$, after which 1,3,2-dioxathiolane 2,2-dioxide (1.05 g, $\left.8.43 \mathrm{mmol}\right)$ in THF (10 mL) was added via cannula. The resulting mixture was stirred at $-78^{\circ} \mathrm{C}$ for 10 minutes, warmed to room temperature, and then refluxed for 12 hours. The reaction mixture was cooled to room temperature, water $(20 \mathrm{~mL})$ was added, and the solution was refluxed for 1 hour. The reaction mixture was then cooled to room temperature and extracted with $\mathrm{Et}_{2} \mathrm{O}(3 \times 100 \mathrm{~mL})$. The organic layer was dried over $\mathrm{MgSO}_{4}$ and concentrated to a yellow liquid, which was purified by flash chromatography $\left(\mathrm{Et}_{2} \mathrm{O}\right)$, furnishing a clear, light-yellow oil (1.27 g, 65\%).

${ }^{1} \mathrm{H}$ NMR (500 MHz, $\left.{ }_{6} \mathrm{D}_{6}\right) \delta 7.32(\mathrm{~d}, 2 \mathrm{H}, \mathrm{J}=8.0), 6.87$ (d, 2H, J=8.0), 3.58 (ddd, 1H, $\mathrm{J}=11.5, \mathrm{~J}=8.5, \mathrm{~J}=3.0), 3.37(\mathrm{~m}, 1 \mathrm{H}), 2.92(\mathrm{dd}, 1 \mathrm{H}, \mathrm{J}=15.5, \mathrm{~J}=9.5), 1.37-1.54(\mathrm{~m}, 2 \mathrm{H}) .{ }^{13} \mathrm{C}$ NMR (125 MHz, $\left.\mathrm{CDCl}_{3}\right) \delta$ 176.7, 140.7, 130.1 (q, J=32.8), 128.6, 126.0 (q, J=3.4), 124.2 (q, $\mathrm{J}=272$ ), 66.7, 45.5, 31.5. FTIR (neat) 2996, 2915, 1772, 1620, 1421, 1375, 1327, $1116 \mathrm{~cm}^{-1}$. HRMS (EI, $m / z)$ calcd. for $\mathrm{C}_{11} \mathrm{H}_{9} \mathrm{~F}_{3} \mathrm{O}_{2}\left(\mathrm{M}^{+}\right) 220.1094$, found 220.1090. bp $145-147^{\circ} \mathrm{C}(0.5$ $\mathrm{mm} \mathrm{Hg}$. 


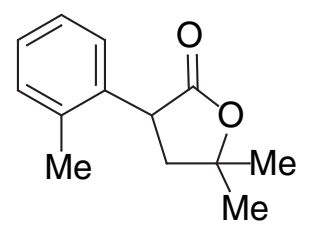

A solution of $n-\mathrm{BuLi}$ (in hexanes; $27.6 \mathrm{~mL}, 73.3 \mathrm{mmol}$ ) was added via syringe to a -78 ${ }^{\circ} \mathrm{C}$ solution of diisopropylamine $(10.3 \mathrm{~mL}, 73.3 \mathrm{mmol})$ in THF $(80 \mathrm{~mL})$. The mixture was stirred at $-78{ }^{\circ} \mathrm{C}$ for 30 minutes and then warmed to $0{ }^{\circ} \mathrm{C}$. A solution of $o$-tolylacetic acid $(5.00 \mathrm{~g}, 33.3 \mathrm{mmol})$ in THF $(20 \mathrm{~mL})$ was then added via cannula. The reaction mixture was warmed to $40{ }^{\circ} \mathrm{C}$ and stirred for 1 hour, and then neat isobutylene oxide ( $3.00 \mathrm{~mL}, 88.1 \mathrm{mmol}$ ) was added via syringe. The resulting solution was stirred at $0{ }^{\circ} \mathrm{C}$ for 1 hour, then refluxed for 18 hours. The solution was cooled to $0{ }^{\circ} \mathrm{C}$, water $(50 \mathrm{~mL}$ ) was added, and the reaction mixture was refluxed for 2 hours. It was then cooled to room temperature, diluted with water $(100 \mathrm{~mL})$, and washed with $\mathrm{Et}_{2} \mathrm{O}(3 \times 50 \mathrm{~mL})$. The aqueous layer was diluted with absolute $\mathrm{EtOH}(50 \mathrm{~mL})$, acidified with $6 \mathrm{~N} \mathrm{HCl}$ (200 $\mathrm{mL}$ ), and refluxed for 2 hours. The reaction mixture was then cooled to room temperature and extracted with $\mathrm{CHCl}_{3}(3 \times 200 \mathrm{~mL})$. The $\mathrm{CHCl}_{3}$ layer was washed with saturated aqueous $\mathrm{NaHCO}_{3}(2 \times 100 \mathrm{~mL})$, dried $\left(\mathrm{Na}_{2} \mathrm{SO}_{4}\right)$, and concentrated. The resulting yellow solid was recrystallized in 2 crops from $\mathrm{Et}_{2} \mathrm{O} /$ pentane (1:10) to afford white crystals, which were collected, washed with pentane, and dried under vacuum (4.14 g, 61\%).

${ }^{1} \mathrm{H}$ NMR (500 MHz, $\left.\mathrm{CDCl}_{3}\right)$ 8 7.17-7.27 (m, 4H), 4.21 (dd, 1H, J=11.0, J=9.0), 2.57 (dd, $1 \mathrm{H}, \mathrm{J}=12.5, \mathrm{~J}=9.5), 2.36(\mathrm{~s}, 3 \mathrm{H}), 2.13(\mathrm{dd}, 1 \mathrm{H}, \mathrm{J}=12.5, \mathrm{~J}=11.5), 1.55(\mathrm{~s}, 3 \mathrm{H}), 1.51(\mathrm{~s}, 3 \mathrm{H}) .{ }^{13} \mathrm{C}$ $\operatorname{NMR}\left(125 \mathrm{MHz}, \mathrm{CDCl}_{3}\right) \delta 177.0,136.4,136.0,130.9,128.0,127.7,126.9,82.4,44.7,43.8$, 29.2, 27.4, 19.9. FTIR $\left(\mathrm{CH}_{2} \mathrm{Cl}_{2}\right) 3019,2983,1750,1653,1497,1457,1377,1278,1144 \mathrm{~cm}^{-1}$. HRMS (EI, $m / z)$ calcd. for $\mathrm{C}_{13} \mathrm{H}_{16} \mathrm{O}_{2}\left(\mathrm{M}^{+}\right)$204.1145, found 204.1136. mp 64-66 ${ }^{\circ} \mathrm{C}$.

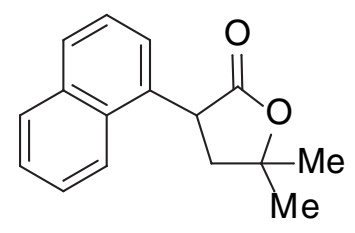


A solution of $n$ - $\mathrm{BuLi}$ (in hexanes; $30.4 \mathrm{~mL}, 80.6 \mathrm{mmol}$ ) was added via syringe to a -78 ${ }^{\circ} \mathrm{C}$ solution of diisopropylamine $(8.15 \mathrm{~mL}, 80.6 \mathrm{mmol})$ in THF $(100 \mathrm{~mL})$. The mixture was stirred at $-78{ }^{\circ} \mathrm{C}$ for 30 minutes and then warmed to $0{ }^{\circ} \mathrm{C}$. A solution of naphthalene-1-acetic acid (5.00 g, $26.9 \mathrm{mmol})$ in THF (20 mL) was then added via cannula, resulting in a bright-orange solution. The reaction mixture was warmed to 50 ${ }^{\circ} \mathrm{C}$ and stirred for 1 hour. Then, neat isobutylene oxide $(2.40 \mathrm{~mL}, 26.9 \mathrm{mmol})$ was added via syringe. The resulting deep-red solution was stirred at $60^{\circ} \mathrm{C}$ for 12 hours. The solution was cooled to $0{ }^{\circ} \mathrm{C}$, diluted with water $(60 \mathrm{~mL})$, and then refluxed for 2 hours. It was then cooled to room temperature, diluted with water $(100 \mathrm{~mL})$, and washed with $\mathrm{Et}_{2} \mathrm{O}(3 \times 50 \mathrm{~mL})$. The aqueous layer was diluted with absolute $\mathrm{EtOH}(75$ $\mathrm{mL})$, acidified with $6 \mathrm{~N} \mathrm{HCl}(50 \mathrm{~mL})$, and refluxed for 2 hours. Then, it was cooled to room temperature and extracted with $\mathrm{CHCl}_{3}(3 \times 200 \mathrm{~mL})$. The $\mathrm{CHCl}_{3}$ layer was successively washed with saturated aqueous $\mathrm{NaHCO}_{3}(2 \times 100 \mathrm{~mL})$ and $\mathrm{NaCl}(1 \times 100$ $\mathrm{mL})$, dried $\left(\mathrm{MgSO}_{4}\right)$, and concentrated. The resulting yellow solid was recrystallized in 2 crops from $\mathrm{CH}_{2} \mathrm{Cl}_{2}$ /pentane (1:5) to afford white crystals, which were collected, washed with pentane, and dried under vacuum $(5.53 \mathrm{~g}, 86 \%)$.

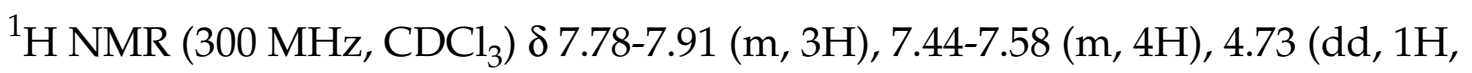
$\mathrm{J}=10.5, \mathrm{~J}=9.6), 2.74(\mathrm{dd}, 1 \mathrm{H}, \mathrm{J}=12.9, \mathrm{~J}=9.3), 2.30(\mathrm{dd}, 1 \mathrm{H}, \mathrm{J}=12.9, \mathrm{~J}=10.5), 1.60(\mathrm{~s}, 3 \mathrm{H}), 1.57$ $(\mathrm{s}, 3 \mathrm{H}) .{ }^{13} \mathrm{C} \mathrm{NMR}\left(125 \mathrm{MHz}, \mathrm{CDCl}_{3}\right) \delta 176.9,134.2,133.8,131.4,129.4,128.5,126.6,126.0$, 125.8, 123.0, 82.7, 44.7, 44.2, 29.2, 27.7. FTIR $\left(\mathrm{CH}_{2} \mathrm{Cl}_{2}\right)$ 3053, 2975, 2932, 1763, 1653, 1512, 1457, 1375, 1268, $1138 \mathrm{~cm}^{-1}$. HRMS (EI, $\mathrm{m} / z$ ) calcd. for $\mathrm{C}_{16} \mathrm{H}_{16} \mathrm{O}_{2}\left(\mathrm{M}^{+}\right) 240.1145$, found 220.1140. mp $122-125^{\circ} \mathrm{C}$.

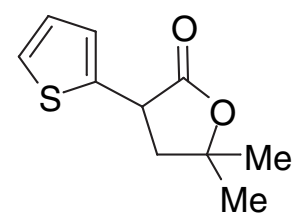

A solution of $n$-BuLi (in hexanes; $38.8 \mathrm{~mL}, 101 \mathrm{mmol}$ ) was added via syringe to a -78 ${ }^{\circ} \mathrm{C}$ solution of diisopropylamine $(14.2 \mathrm{~mL}, 101 \mathrm{mmol})$ in THF $(150 \mathrm{~mL})$. The mixture 
was stirred at $-78{ }^{\circ} \mathrm{C}$ for 30 minutes and then warmed to $0{ }^{\circ} \mathrm{C}$. A solution of thiophene2-acetic acid (7.00 g, $49.2 \mathrm{mmol})$ in THF (50 mL) was added via cannula, and the mixture was warmed to $40{ }^{\circ} \mathrm{C}$ and stirred for 1 hour. Neat isobutylene oxide (4.44 mL, 49.2 mmol) was added via syringe, and the resulting solution was refluxed for 18 hours. Water $(100 \mathrm{~mL})$ was then added, and the reaction mixture was refluxed for 2 hours. It was then cooled to room temperature and washed with $\mathrm{Et}_{2} \mathrm{O}(3 \times 150 \mathrm{~mL})$. The aqueous layer was diluted with absolute $\mathrm{EtOH}(100 \mathrm{~mL})$, acidified with $1 \mathrm{~N} \mathrm{HCl}(75 \mathrm{~mL})$, and refluxed for 2 hours. It was then cooled to room temperature and extracted with $\mathrm{CHCl}_{3}(3 \times 200 \mathrm{~mL})$. The $\mathrm{CHCl}_{3}$ layer was washed successively with saturated aqueous $\mathrm{NaHCO}_{3}(2 \times 100 \mathrm{~mL})$ and $\mathrm{NaCl}(2 \times 100 \mathrm{~mL})$, dried $\left(\mathrm{MgSO}_{4}\right)$, and concentrated. The resulting tan solid was recrystallized in 2 crops from $\mathrm{CH}_{2} \mathrm{Cl}_{2}$ / pentane (3:10) to afford light-tan crystals, which were collected, washed with pentane, and dried under vacuum $(8.60 \mathrm{~g}, 89 \%)$.

${ }^{1} \mathrm{H}$ NMR $\left(300 \mathrm{MHz}, \mathrm{CDCl}_{3}\right) \delta 7.25(\mathrm{dd}, 1 \mathrm{H}, \mathrm{J}=5.1, \mathrm{~J}=1.2), 6.97-7.03(\mathrm{~m}, 2 \mathrm{H}), 4.27$ (ddd, $1 \mathrm{H}, \mathrm{J}=12.6, \mathrm{~J}=11.7, \mathrm{~J}=1.2), 2.67$ (dd, 1H, J=12.6, J=9.0), 2.34 (dd, 1H, J=12.9, J=11.4), 1.54 (s, $3 \mathrm{H}), 1.49(\mathrm{~s}, 3 \mathrm{H}) .{ }^{13} \mathrm{C} \mathrm{NMR}\left(125 \mathrm{MHz}, \mathrm{CDCl}_{3}\right) \delta 175.0,138.6,126.9,125.6,124.9,82.5$, 43.7, 42.1, 28.7, 26.9. FTIR $\left(\mathrm{CH}_{2} \mathrm{Cl}_{2}\right) 3102,2972,2931,2870,1759,1653,1456,1375,1300$, $1139 \mathrm{~cm}^{-1}$. HRMS (EI, $\mathrm{m} / z$ ) calcd. for $\mathrm{C}_{10} \mathrm{H}_{12} \mathrm{O}_{2} \mathrm{~S}\left(\mathrm{M}^{+}\right)$196.0553, found 196.0561. mp 61$63{ }^{\circ} \mathrm{C}$.

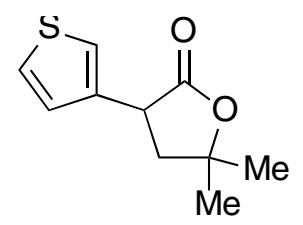

A solution of $n$ - $\mathrm{BuLi}$ (in hexanes; $16.7 \mathrm{~mL}, 44.3 \mathrm{mmol}$ ) was added via syringe to a -78 ${ }^{\circ} \mathrm{C}$ solution of diisopropylamine $(6.21 \mathrm{~mL}, 44.3 \mathrm{mmol})$ in THF $(70 \mathrm{~mL})$. The mixture was stirred at $-78{ }^{\circ} \mathrm{C}$ for 30 minutes and then warmed to $0{ }^{\circ} \mathrm{C}$. A solution of thiophene-3acetic acid $(3.00 \mathrm{~g}, 21.1 \mathrm{mmol})$ in THF $(10 \mathrm{~mL})$ was added via cannula, and the reaction mixture was warmed to $50{ }^{\circ} \mathrm{C}$ and stirred for 40 minutes. Neat isobutylene oxide (1.90 $\mathrm{mL}, 21.1 \mathrm{mmol}$ ) was added via syringe, and the resulting solution was stirred at $50{ }^{\circ} \mathrm{C}$ 
for 12 hours. Water $(100 \mathrm{~mL})$ was added, and the reaction mixture was refluxed for 2 hours. It was then cooled to room temperature and washed with $\mathrm{Et}_{2} \mathrm{O}(3 \times 150 \mathrm{~mL})$. The aqueous layer was diluted with absolute $\mathrm{EtOH}(50 \mathrm{~mL})$, acidified with $2 \mathrm{~N} \mathrm{HCl}(75$ $\mathrm{mL}$ ), and refluxed for 2 hours. Then, it was cooled to room temperature and extracted with $\mathrm{CHCl}_{3}(4 \times 200 \mathrm{~mL})$. The $\mathrm{CHCl}_{3}$ layer was washed with saturated aqueous $\mathrm{NaHCO}_{3}(2 \times 100 \mathrm{~mL})$, dried $\left(\mathrm{Na}_{2} \mathrm{SO}_{4}\right)$, and concentrated to afford a gold oil, which was purified by flash chromatography ( $25 \% \mathrm{Et}_{2} \mathrm{O} / 75 \%$ pentane $\ddagger 40 \% \mathrm{Et}_{2} \mathrm{O} / 60 \%$ pentane) to provide a clear, light-yellow oil ( $2.91 \mathrm{~g}, 70 \%)$.

${ }^{1} \mathrm{H}$ NMR $\left(300 \mathrm{MHz}, \mathrm{CDCl}_{3}\right) \delta 7.34(\mathrm{dd}, 1 \mathrm{H}, \mathrm{J}=5.1, \mathrm{~J}=2.7), 7.23-7.26(\mathrm{~m}, 1 \mathrm{H}), 7.09(\mathrm{dd}$, $1 \mathrm{H}, \mathrm{J}=5.1, \mathrm{~J}=1.2), 4.12(\mathrm{dd}, 1 \mathrm{H}, \mathrm{J}=11.4, \mathrm{~J}=9.0), 2.58(\mathrm{dd}, 1 \mathrm{H}, \mathrm{J}=12.6, \mathrm{~J}=9.0), 2.25$ (app t, 1H, $\mathrm{J}=12.0), 1.52(\mathrm{~s}, 3 \mathrm{H}), 1.48(\mathrm{~s}, 3 \mathrm{H}) .{ }^{13} \mathrm{C} \mathrm{NMR}\left(125 \mathrm{MHz}, \mathrm{CDCl}_{3}\right) \delta 176.0,136.7,127.0,126.3$, 122.1, 82.4, 42.9, 42.1, 28.8, 26.9. FTIR (neat) 3105, 2977, 2934, 1762, 1456, 1375, 1261, 1140 $\mathrm{cm}^{-1}$. HRMS (EI, $\left.m / z\right)$ calcd. for $\mathrm{C}_{10} \mathrm{H}_{12} \mathrm{O}_{2} \mathrm{~S}\left(\mathrm{M}^{+}\right)$196.0553, found 196.0557 . 


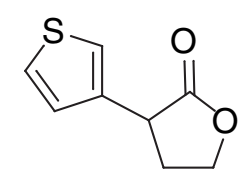

A solution of $n$-BuLi (in hexanes; $16.0 \mathrm{~mL}, 42.3 \mathrm{mmol}$ ) was added via syringe to a -78 ${ }^{\circ} \mathrm{C}$ solution of diisopropylamine $(5.93 \mathrm{~mL}, 42.3 \mathrm{mmol})$ in THF $(20 \mathrm{~mL})$. The mixture was stirred at $-78{ }^{\circ} \mathrm{C}$ for 45 minutes, and then a solution of thiophene-3-acetic acid ( $2.86 \mathrm{~g}$, $20.1 \mathrm{mmol})$ in THF $(20 \mathrm{~mL})$ was added via cannula. The mixture was stirred for 20 minutes at $-78{ }^{\circ} \mathrm{C}$, warmed to room temperature, and stirred for 45 minutes. A solution of 1,3,2-dioxathiolane 2,2-dioxide ( $2.50 \mathrm{~g}, 20.1 \mathrm{mmol})$ in THF ( $20 \mathrm{~mL})$ was added via syringe. 1,2-Dimethoxyethane $(15 \mathrm{~mL})$ was added, and the resulting solution was refluxed for 16 hours. Water $(20 \mathrm{~mL})$ was then added, and the resulting mixture was extracted with $\mathrm{CH}_{2} \mathrm{Cl}_{2}(3 \times 100 \mathrm{~mL})$. The organic layer was washed successively with saturated aqueous $\mathrm{NaHCO}_{3}(2 \times 100 \mathrm{~mL})$ and $\mathrm{NaCl}(1 \times 50 \mathrm{~mL})$, and then it was concentrated, affording a yellow liquid. Purification by flash chromatography (50\% $\mathrm{Et}_{2} \mathrm{O} / 50 \%$ pentane $\$ 75 \% \mathrm{Et}_{2} \mathrm{O} / 25 \%$ pentane) provided a clear, light-yellow oil (1.86 g, $55 \%)$.

${ }^{1} \mathrm{H}$ NMR $\left(300 \mathrm{MHz}, \mathrm{CDCl}_{3}\right) \delta 7.35(\mathrm{dd}, 1 \mathrm{H}, \mathrm{J}=4.8, \mathrm{~J}=3.0), 7.24-7.26(\mathrm{~m}, 1 \mathrm{H}), 7.10(\mathrm{dd}$, $1 \mathrm{H}, \mathrm{J}=4.8, \mathrm{~J}=1.2$ ), 4.47 (ddd, 1H, J=12.3, J=8.7, J=3.6), 4.35 (ddd, 1H, J=15.6, J=9.0, J=6.6), $3.91(\mathrm{dd}, 1 \mathrm{H}, \mathrm{J}=9.6, \mathrm{~J}=9.0)$, 2.68-2.79 (m, 1H), 2.40-2.53 (m, 1H). ${ }^{13} \mathrm{C}$ NMR $(125 \mathrm{MHz}$, $\left.\mathrm{CDCl}_{3}\right) \delta 177.0,136.3,126.9,126.4,122.1,66.7,40.7,30.6$. FTIR (neat) 3103, 2990, 2910, $1772,1482,1373,1156,1024 \mathrm{~cm}^{-1}$. HRMS (ESI, $\left.m / z\right)$ calcd. for $\mathrm{C}_{8} \mathrm{H}_{8} \mathrm{O}_{2} \mathrm{~S}(\mathrm{M}+\mathrm{Na})^{+}$ 191.0137, found 191.0141. 


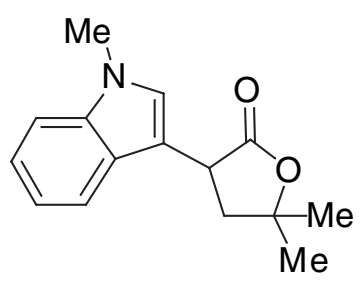

A solution of $n$ - $\mathrm{BuLi}$ (in hexanes; $19.6 \mathrm{~mL}, 58.1 \mathrm{mmol}$ ) was added via syringe to a -78 ${ }^{\circ} \mathrm{C}$ solution of diisopropylamine $(8.15 \mathrm{~mL}, 58.1 \mathrm{mmol})$ in THF $(50 \mathrm{~mL})$. The mixture was stirred at $-78{ }^{\circ} \mathrm{C}$ for 40 minutes, and then a solution of 1-methyl-indole-3-acetic acid $(5.00 \mathrm{~g}, 26.4 \mathrm{mmol})$ in THF $(20 \mathrm{~mL})$ was added via cannula. The mixture was stirred at $-78^{\circ} \mathrm{C}$ for 75 minutes, and then neat isobutylene oxide $(2.38 \mathrm{~mL}, 26.4 \mathrm{mmol})$ was added via syringe. The resulting solution was warmed to room temperature and stirred for 12 hours. Water $(100 \mathrm{~mL})$ was then added, and the resulting solution was refluxed for 2 hours. It was then cooled to room temperature, washed with $\mathrm{Et}_{2} \mathrm{O}(3 \times 150 \mathrm{~mL})$, diluted with water $(160 \mathrm{~mL})$ and absolute $\mathrm{EtOH}(50 \mathrm{~mL})$, and acidified with $6 \mathrm{~N} \mathrm{HCl}(70 \mathrm{~mL})$. This reaction mixture was refluxed for 4 hours and then cooled to room temperature and extracted with $\mathrm{CHCl}_{3}(3 \times 200 \mathrm{~mL})$. The $\mathrm{CHCl}_{3}$ layer was successively washed with saturated aqueous $\mathrm{NaHCO}_{3}(3 \times 100 \mathrm{~mL})$ and water $(2 \times 100 \mathrm{~mL})$, dried $\left(\mathrm{Na}_{2} \mathrm{SO}_{4}\right)$, and concentrated. The resulting brown solid was recrystallized in 2 crops from $\mathrm{EtOH} / \mathrm{H}_{2} \mathrm{O}$ (1:1) to afford dark-tan crystals, which were collected, washed with pentane, and dried under vacuum $(5.09 \mathrm{~g}, 79 \%)$.

${ }^{1} \mathrm{H}$ NMR (300 MHz, $\left.\mathrm{C}_{6} \mathrm{D}_{6}\right) \delta 7.54(\mathrm{~d}, 1 \mathrm{H}, \mathrm{J}=7.2), 7.16-7.29(\mathrm{~m}, 2 \mathrm{H}), 7.03(\mathrm{~d}, 1 \mathrm{H}, \mathrm{J}=7.8)$, $6.95(\mathrm{~s}, 1 \mathrm{H}), 4.00(\mathrm{dd}, 1 \mathrm{H}, \mathrm{J}=10.2, \mathrm{~J}=9.9), 2.91$ (s, 3H), 2.11 (dd, 1H, J=12.3, J=9.3), 1.87 (dd, $1 \mathrm{H}, \mathrm{J}=12.3, \mathrm{~J}=11.1), 1.10(\mathrm{~s}, 3 \mathrm{H}), 1.02(\mathrm{~s}, 3 \mathrm{H}) .{ }^{13} \mathrm{C} \mathrm{NMR}\left(125 \mathrm{MHz}, \mathrm{CDCl}_{3}\right) \delta$ 177.2, 137.4, 127.1, 126.7, 122.0, 119.4, 118.9, 110.0, 109.7, 82.5, 43.5, 38.9, 38.8, 29.1, 27.2. FTIR $\left(\mathrm{CH}_{2} \mathrm{Cl}_{2}\right) 3055,2975,2934,1770,1653,1558,1474,1375,1256,1140 \mathrm{~cm}^{-1}$. HRMS (EI, $\left.m / z\right)$ calcd. for $\mathrm{C}_{15} \mathrm{H}_{17} \mathrm{NO}_{2}\left(\mathrm{M}^{+}\right) 243.1254$, found 243.1255. mp 121-122 ${ }^{\circ} \mathrm{C}$.<smiles>CCCOC(=O)C(CC)c1ccccc1</smiles> 
Oxalyl chloride $(10.6 \mathrm{~mL}, 122 \mathrm{mmol})$ was added over 5 minutes to a $-78^{\circ} \mathrm{C}$ solution of 2-phenylbutyric acid ( $8.00 \mathrm{~g}, 48.7 \mathrm{mmol})$ and $N, N$ dimethylformamide (3 drops) in $\mathrm{CH}_{2} \mathrm{Cl}_{2}(40 \mathrm{~mL})$. The resulting mixture was stirred at $-78{ }^{\circ} \mathrm{C}$ for 2 hours, and then it was warmed to room temperature and concentrated. $\mathrm{CH}_{2} \mathrm{Cl}_{2}(40 \mathrm{~mL})$ was then added to the unpurified acid chloride, and the resulting solution was cooled to $0^{\circ} \mathrm{C}$. To this solution was added 2-methyl-2-propanol $(5.13 \mathrm{~mL}, 53.6 \mathrm{mmol})$ and then $\mathrm{NEt}_{3}(3 \mathrm{~mL})$. The reaction mixture was slowly warmed to room temperature and then stirred for 15 hours. Saturated $\mathrm{NH}_{4} \mathrm{Cl}(50 \mathrm{~mL})$ was added, and then the reaction mixture was extracted with $\mathrm{CH}_{2} \mathrm{Cl}_{2}(3 \times 50 \mathrm{~mL})$. The combined organic layers were washed with saturated $\mathrm{NaHCO}_{3}(2 \times 100 \mathrm{~mL})$ and water $(2 \times 50 \mathrm{~mL})$, dried over $\mathrm{MgSO}_{4}$, filtered, and concentrated, to yield an orange oil. Purification by vacuum distillation $\left(83-85{ }^{\circ} \mathrm{C}, 1\right.$ $\mathrm{mm} \mathrm{Hg}$ ) furnished the target compound as a clear, colorless liquid $(6.06 \mathrm{~g}, 52 \%)$.

${ }^{1} \mathrm{H}$ NMR $\left(500 \mathrm{MHz}, \mathrm{C}_{6} \mathrm{D}_{6}\right) \delta 7.32(\mathrm{dd}, 2 \mathrm{H}, \mathrm{J}=6.0, \mathrm{~J}=0.5), 7.13(\mathrm{~m}, 2 \mathrm{H}), 7.04(\mathrm{tt}, 1 \mathrm{H}$, $\mathrm{J}=7.5, \mathrm{~J}=1.5), 3.35(\mathrm{t}, 1 \mathrm{H}, \mathrm{J}=7.5), 2.12(\mathrm{~d}$ app t $, 1 \mathrm{H}, \mathrm{J}=13.5, \mathrm{~J}=7.5), 1.70(\mathrm{~d}$ app t, 1H, J=13.5, $\mathrm{J}=7.5), 1.29$ (s, 9H), 0.83 (app t, 3H J=7.5). ${ }^{13} \mathrm{C} \mathrm{NMR}\left(125 \mathrm{MHz}, \mathrm{CDCl}_{3}\right) \delta 173.5,139.9$, 128.5, 128.0, 127.0, 80.6, 54.6, 28.1, 27.0, 12.4. FTIR (thin film) 3030, 2969, 2933, 2876, $1729,1454,1148,1078,749,698 \mathrm{~cm}^{-1}$. 


\section{Preparation of Silyl Ketene Acetals}

These reactions have not been optimized.

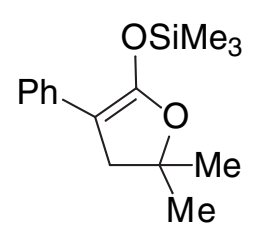

General procedure. A solution of $n$-BuLi (in hexanes; $9.00 \mathrm{~mL}, 22.1 \mathrm{mmol}$ ) was added via syringe to a $-78{ }^{\circ} \mathrm{C}$ solution of diisopropylamine $(3.10 \mathrm{~mL}, 22.1 \mathrm{mmol})$ in THF $(10 \mathrm{~mL})$. The mixture was stirred at $-78^{\circ} \mathrm{C}$ for 45 minutes, and then a solution of the lactone $(4.0 \mathrm{~g}, 21.0 \mathrm{mmol})$ in THF $(10 \mathrm{~mL})$ was added via cannula. The mixture was stirred at $-78{ }^{\circ} \mathrm{C}$ for 60 minutes, and then $\mathrm{Me}_{3} \mathrm{SiCl}(2.80 \mathrm{~mL}, 22.1 \mathrm{mmol})$ was added, resulting in a clear, light-yellow solution which was warmed to room temperature and stirred for 3 hours. The THF was then removed, and the residue was taken up in pentane and filtered. The solvent was removed, and the yellow liquid was purified by fractional distillation $\left(126-128^{\circ} \mathrm{C}, 0.5 \mathrm{~mm} \mathrm{Hg}\right)$ to afford a clear, colorless liquid ( $2.01 \mathrm{~g}$, $37 \%)$.

${ }^{1} \mathrm{H}$ NMR $\left(300 \mathrm{MHz}, \mathrm{C}_{6} \mathrm{D}_{6}\right) \delta$ 7.70-7.75 (m, 2H), 7.43-7.49 (m, 2H), 7.14-7.19 (m, 1H), $2.67(\mathrm{~s}, 2 \mathrm{H}), 1.29(\mathrm{~s}, 6 \mathrm{H}), 0.35(\mathrm{~s}, 9 \mathrm{H}) .{ }^{13} \mathrm{C} \mathrm{NMR}\left(125 \mathrm{MHz}, \mathrm{C}_{6} \mathrm{D}_{6}\right) \delta$ 153.8, 137.3, 129.5, 128.8, 124.3, 123.3, 82.0, 43.9, 29.2, 1.0. FTIR $\left(\mathrm{CH}_{2} \mathrm{Cl}_{2}\right) 2900,2849,1662,1659,1600,1502$, $1461,1371,1170,1161,1068,1004,983,857 \mathrm{~cm}^{-1}$. bp $126-128^{\circ} \mathrm{C}(0.5 \mathrm{~mm} \mathrm{Hg})$. 


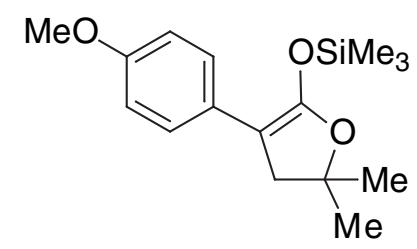

The general procedure was followed, using $n$-BuLi (in hexanes; $3.40 \mathrm{~mL}, 9.53 \mathrm{mmol}$ ), diisopropylamine (0.965 mL, $9.53 \mathrm{mmol})$, lactone ( $2.00 \mathrm{~g}$, $9.08 \mathrm{mmol})$, and $\mathrm{Me}_{3} \mathrm{SiCl}(1.25$ $\mathrm{mL}, 9.53 \mathrm{mmol})$ in THF $(25 \mathrm{~mL})$. Purification by chromatography on Florisil (pentane) inside a glove box afforded a white crystalline solid (1.98 g, 75\%).

${ }^{1} \mathrm{H}$ NMR (300 MHz, $\left.\mathrm{C}_{6} \mathrm{D}_{6}\right) 7.25(\mathrm{dd}, 2 \mathrm{H}, \mathrm{J}=6.9, \mathrm{~J}=2.1), 6.81(\mathrm{dd}, 2 \mathrm{H}, \mathrm{J}=6.9, \mathrm{~J}=2.1), 3.77$ (s, 3H), 1.43 (s, 6H), 0.33 (s, 9H). ${ }^{13} \mathrm{C}$ NMR (125 MHz, $\left.\mathrm{C}_{6} \mathrm{D}_{6}\right) \delta 156.2,152.2,129.8,125.1$, 114.1, 81.6, 81.4, 55.7, 43.9, 29.0, 0.7. FTIR $\left(\mathrm{CH}_{2} \mathrm{Cl}_{2}\right)$ 3152, 3007, 2899, 1669, 1666, 1514, 1369, 1240, 1168, 1093, $991 \mathrm{~cm}^{-1}$. HRMS (EI, m/z) calcd. for $\mathrm{C}_{16} \mathrm{H}_{24} \mathrm{O}_{3} \mathrm{Si}\left(\mathrm{M}^{+}\right)$292.1489, found 292.1480. $\mathrm{mp} 54-56^{\circ} \mathrm{C}$.

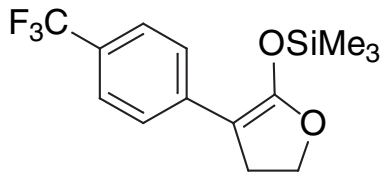

The general procedure was followed, using n-BuLi (in hexanes; $2.30 \mathrm{~mL}, 6.48 \mathrm{mmol}$ ), diisopropylamine $(0.910 \mathrm{~mL}, 6.48 \mathrm{mmol})$, lactone $(1.42 \mathrm{~g}, 6.17 \mathrm{mmol})$, and $\mathrm{Me}_{3} \mathrm{SiCl}$ $(0.822 \mathrm{~mL}, 6.48 \mathrm{mmol})$ in THF $(30 \mathrm{~mL})$. Purification by chromatography on Florisil (pentane) inside a glove box afforded a white crystalline solid $(1.21 \mathrm{~g}, 65 \%)$.

${ }^{1} \mathrm{H}$ NMR (300 MHz, $\left.\mathrm{C}_{6} \mathrm{D}_{6}\right) 7.51(\mathrm{dd}, 2 \mathrm{H}, \mathrm{J}=9.0, \mathrm{~J}=0.9), 7.37$ (dd, 2H, J=9.0, J=0.9), 3.80 $(\mathrm{t}, 2 \mathrm{H}, \mathrm{J}=8.4), 2.40(\mathrm{t}, 2 \mathrm{H}, \mathrm{J}=8.1), 0.20(\mathrm{~s}, 9 \mathrm{H}) .{ }^{13} \mathrm{C} \mathrm{NMR}\left(125 \mathrm{MHz}, \mathrm{CD}_{2} \mathrm{Cl}_{2}\right) \delta$ 157.9, 140.7, 129.2, 125.5 (q, J=4.0), 124.4 (q, J=32.4), 123.9, 82.4, 67.6, 30.4, 0.7. FTIR $\left(\mathrm{CH}_{2} \mathrm{Cl}_{2}\right)$ 3152, $3008,1658,1607,1529,1327,1192,1121,1067,990 \mathrm{~cm}^{-1}$. HRMS (EI, $\mathrm{m} / \mathrm{z}$ ) calcd. for $\mathrm{C}_{14} \mathrm{H}_{17} \mathrm{~F}_{3} \mathrm{O}_{2} \mathrm{Si}\left(\mathrm{M}^{+}\right) 302.0944$, found 302.0944. $\mathrm{mp} 43-44{ }^{\circ} \mathrm{C}$. 


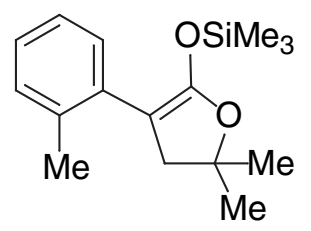

The general procedure was followed, using $n$-BuLi (in hexanes; $1.39 \mathrm{~mL}, 3.89 \mathrm{mmol}$ ), diisopropylamine $(0.545 \mathrm{~mL}, 3.89 \mathrm{mmol})$, lactone $(0.795 \mathrm{~g}, 3.89 \mathrm{mmol})$, and $\mathrm{Me}_{3} \mathrm{SiCl}$ (0.494 mL, $3.89 \mathrm{mmol})$ in THF (22 mL). Purification by chromatography on Florisil (pentane) inside a glove box afforded a clear, colorless oil $(0.865 \mathrm{~g}, 81 \%)$.

${ }^{1} \mathrm{H}$ NMR $\left(300 \mathrm{MHz}, \mathrm{C}_{6} \mathrm{D}_{6}\right)$ 反 7.30-7.33 (m, 1H), 6.99-7.15 (m, 3H), $2.62(\mathrm{~s}, 2 \mathrm{H}), 2.56(\mathrm{~s}$, $3 \mathrm{H}), 1.22(\mathrm{~s}, 6 \mathrm{H}), 0.10(\mathrm{~s}, 9 \mathrm{H}) .{ }^{13} \mathrm{C}$ NMR $\left(125 \mathrm{MHz}, \mathrm{C}_{6} \mathrm{D}_{6}\right) \delta$ 152.1, 136.9, 136.1, 131.0, 129.5, 126.1, 126.0, 82.7, 81.4, 46.7, 28.6, 21.6, 0.7. FTIR $\left(\mathrm{CH}_{2} \mathrm{Cl}_{2}\right)$ 2971, 1688, 1679, 1598, $1461,1354,1169,1084,994,857 \mathrm{~cm}^{-1}$. HRMS (ESI, $\left.m / z\right)$ calcd. for $\mathrm{C}_{16} \mathrm{H}_{24} \mathrm{O}{ }_{2} \mathrm{Si}(\mathrm{M}+\mathrm{H})^{+}$ 277.1618, found 277.1624.

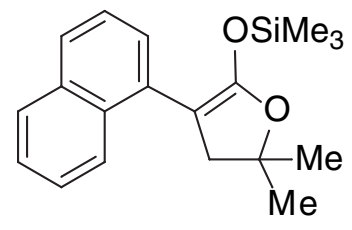

The general procedure was followed, using $n$-BuLi (in hexanes; $1.78 \mathrm{~mL}, 4.98 \mathrm{mmol}$ ), diisopropylamine $(0.698 \mathrm{~mL}, 4.98 \mathrm{mmol})$, lactone $(1.14 \mathrm{~g}, 4.74 \mathrm{mmol})$, and $\mathrm{Me}_{3} \mathrm{SiCl}$ (0.632 mL, $4.98 \mathrm{mmol})$ in THF $(25 \mathrm{~mL})$. Purification by chromatography on Florisil (pentane) inside a glove box afforded a white solid (0.709 $\mathrm{g}, 48 \%)$.

${ }^{1} \mathrm{H}$ NMR $\left(300 \mathrm{MHz}, \mathrm{C}_{6} \mathrm{D}_{6}\right) \delta 8.31(\mathrm{ddt}, 1 \mathrm{H}, \mathrm{J}=8.1, \mathrm{~J}=0.1), 7.66$ (ddt, $1 \mathrm{H}, \mathrm{J}=8.1, \mathrm{~J}=0.6$ ), $7.53(\mathrm{~d}, 1 \mathrm{H}, \mathrm{J}=8.1), 7.24-7.39(\mathrm{~m}, 4 \mathrm{H}), 2.76(\mathrm{~s}, 2 \mathrm{H}), 1.27(\mathrm{~s}, 6 \mathrm{H}), 0.00(\mathrm{~s}, 9 \mathrm{H}) .{ }^{13} \mathrm{C}$ NMR $(75$ $\left.\mathrm{MHz}, \mathrm{C}_{6} \mathrm{D}_{6}\right) \delta 152.9,135.2,135.0,132.7,129.1,127.9,126.7,126.3,126.11,126.06,125.4$, 81.8, 81.6, 47.3, 28.7, 0.7. FTIR $\left(\mathrm{CH}_{2} \mathrm{Cl}_{2}\right) 3054,2985,1558,1540,1506,1456,1420,1265$, $856 \mathrm{~cm}^{-1}$. HRMS (EI, $\mathrm{m} / \mathrm{z}$ ) calcd. for $\mathrm{C}_{19} \mathrm{H}_{24} \mathrm{O}_{2} \mathrm{Si}\left(\mathrm{M}^{+}\right)$312.1540, found 312.1549. mp 86$89^{\circ} \mathrm{C}$. 


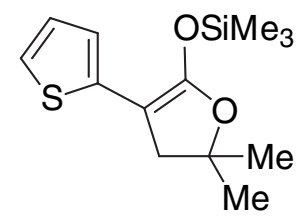

A solution of $n$-BuLi (in hexanes; $2.78 \mathrm{~mL}, 7.79 \mathrm{mmol}$ ) was added via syringe to a -78 ${ }^{\circ} \mathrm{C}$ solution of diisopropylamine $(1.10 \mathrm{~mL}, 7.79 \mathrm{mmol})$ in THF $(15 \mathrm{~mL})$. The mixture was stirred at $-78{ }^{\circ} \mathrm{C}$ for 45 minutes, and then a solution of lactone $(1.53 \mathrm{~g}, 7.79 \mathrm{mmol})$ in THF $(10 \mathrm{~mL})$ was added via cannula. The mixture was stirred at $-78^{\circ} \mathrm{C}$ for 60 minutes, after which $\mathrm{Me}_{3} \mathrm{SiCl}(0.988 \mathrm{~mL}, 7.79 \mathrm{mmol})$ was added, resulting in a clear, light-yellow solution, which was warmed to room temperature and stirred for 3 hours. The THF was then removed, and the yellow residue was taken up in pentane and filtered. The pentane was removed, and the yellow residue was taken into a glove box and purified by chromatography on activated Florisil (pentane), which afforded a clear, colorless oil $(1.02 \mathrm{~g}, 49 \%)$.

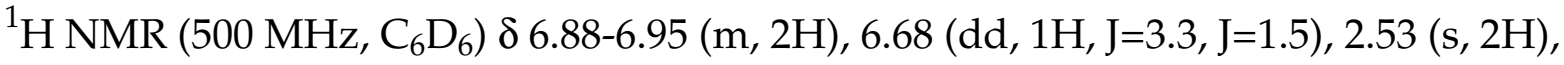
$1.14(\mathrm{~s}, 6 \mathrm{H}), 0.27$ (s, 9H). ${ }^{13} \mathrm{C}$ NMR $\left(75 \mathrm{MHz}, \mathrm{C}_{6} \mathrm{D}_{6}\right) \delta 152.8,140.3,127.1,120.8,118.8$, 82.8, 81.3, 44.2, 28.8, 0.8. FTIR $\left(\mathrm{CH}_{2} \mathrm{Cl}_{2}\right) 2963,2901,2851,1678,1665,1520,1461,1369$, $1238,1166,1091,1043,939 \mathrm{~cm}^{-1}$. HRMS (ESI, $\mathrm{m} / \mathrm{z}$ ) calcd. for $\mathrm{C}_{13} \mathrm{H}_{20} \mathrm{O}_{2} \mathrm{SSi}(\mathrm{M}+\mathrm{H})^{+}$ 269.1026, found 269.1030. 


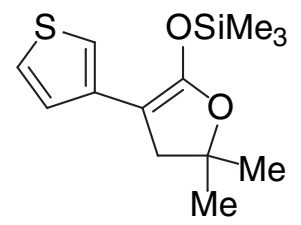

The general procedure was followed, using n-BuLi (in hexanes; $2.17 \mathrm{~mL}, 5.76 \mathrm{mmol}$ ), diisopropylamine $(0.807 \mathrm{~mL}, 5.76 \mathrm{mmol})$, lactone $(1.13 \mathrm{~g}, 5.76 \mathrm{mmol})$, and $\mathrm{Me}_{3} \mathrm{SiCl}$ (0.731 mL, $5.76 \mathrm{mmol})$ in THF $(25 \mathrm{~mL})$. Purification by chromatography on Florisil (pentane) inside a glove box afforded a clear, colorless oil ( $0.824 \mathrm{~g}, 53 \%)$.

${ }^{1} \mathrm{H}$ NMR $\left(300 \mathrm{MHz}, \mathrm{C}_{6} \mathrm{D}_{6}\right) \delta 7.57(\mathrm{dd}, 1 \mathrm{H}, \mathrm{J}=5.1, \mathrm{~J}=1.2), 7.08(\mathrm{dd}, 1 \mathrm{H}, \mathrm{J}=5.4, \mathrm{~J}=3.0), 6.68$ $(\mathrm{dd}, 1 \mathrm{H}, \mathrm{J}=2.7, \mathrm{~J}=1.2), 2.48(\mathrm{~s}, 2 \mathrm{H}), 1.18(\mathrm{~s}, 6 \mathrm{H}), 0.22(\mathrm{~s}, 9 \mathrm{H}) .{ }^{13} \mathrm{C} \mathrm{NMR}\left(75 \mathrm{MHz}, \mathrm{C}_{6} \mathrm{D}_{6}\right) \delta$ 152.5, 138.0, 126.1, 125.3, 114.1, 82.0, 80.7, 44.1, 28.9, 0.8. FTIR $\left(\mathrm{CH}_{2} \mathrm{Cl}_{2}\right)$ 2962, 2900, 2849, $1689,1679,1527,1461,1382,1370,1342,1228,1198,1163,1098,1016,914 \mathrm{~cm}^{-1}$. HRMS (EI, $m / z$ ) calcd. for $\mathrm{C}_{13} \mathrm{H}_{20} \mathrm{O}_{2} \mathrm{SSi}\left(\mathrm{M}^{+}\right)$268.0948, found 268.0941.

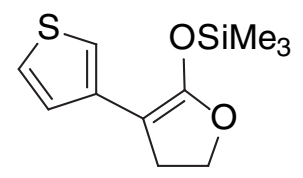

A solution of $n$-BuLi (in hexanes; $7.24 \mathrm{~mL}, 17.8 \mathrm{mmol}$ ) was added via syringe to a -78 ${ }^{\circ} \mathrm{C}$ solution of diisopropylamine $(2.49 \mathrm{~mL}, 17.8 \mathrm{mmol})$ in THF $(20 \mathrm{~mL})$. The mixture was stirred at $-78{ }^{\circ} \mathrm{C}$ for 45 minutes, after which a solution of the lactone $(2.85 \mathrm{~g}, 16.9 \mathrm{mmol})$ in THF $(15 \mathrm{~mL})$ was added via cannula. The mixture was stirred at $-78^{\circ} \mathrm{C}$ for 60 minutes, and then $\mathrm{Me}_{3} \mathrm{SiCl}(2.26 \mathrm{~mL}, 17.8 \mathrm{mmol})$ was added, resulting in a clear, lightyellow solution, which was warmed to room temperature and stirred for 3 hours. The THF was then removed, and the orange residue was taken up in pentane and filtered. The solvent was removed, and the orange liquid was purified by fractional distillation $\left(127^{\circ} \mathrm{C}, 1.0 \mathrm{~mm} \mathrm{Hg}\right)$, which afforded a clear, colorless oil $(1.50 \mathrm{~g}, 45 \%)$.

${ }^{1} \mathrm{H}$ NMR (500 MHz, $\left.\mathrm{C}_{6} \mathrm{D}_{6}\right) \delta 7.57(\mathrm{dd}, 1 \mathrm{H}, \mathrm{J}=5.0, \mathrm{~J}=1.0), 7.07(\mathrm{dd}, 1 \mathrm{H}, \mathrm{J}=5.0, \mathrm{~J}=3.0), 6.66$ $(\mathrm{dd}, 1 \mathrm{H}, \mathrm{J}=3.0, \mathrm{~J}=1.5), 3.87(\mathrm{t}, 2 \mathrm{H}, \mathrm{J}=9.0), 2.52(\mathrm{t}, 2 \mathrm{H}, \mathrm{J}=8.0), 0.22(\mathrm{~s}, 9 \mathrm{H}) .{ }^{13} \mathrm{C}$ NMR $(125$ $\left.\mathrm{MHz}, \mathrm{C}_{6} \mathrm{D}_{6}\right) \delta 137.5,128.7,126.0,125.5,114.5,81.2,67.1,31.1,0.7$. FTIR $\left(\mathrm{CH}_{2} \mathrm{Cl}_{2}\right) 3008$, 
2961, 2902, 2856, 1688, 1679, 1528, 1365, 1327, 1254, $1191 \mathrm{~cm}^{-1}$. HRMS (ESI, m/z) calcd. for $\mathrm{C}_{11} \mathrm{H}_{16} \mathrm{O}_{2} \mathrm{SSi}(\mathrm{M}+\mathrm{H})^{+} 241.0713$, found 241.0713. bp 127-128 ${ }^{\circ} \mathrm{C}(1.0 \mathrm{~mm} \mathrm{Hg})$.

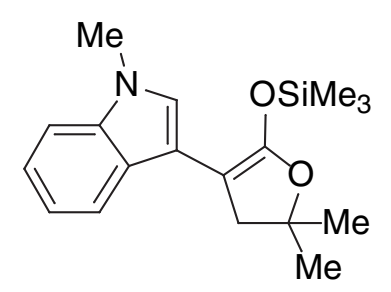

The general procedure was followed, using $n$-BuLi (in hexanes; $1.03 \mathrm{~mL}, 2.89 \mathrm{mmol}$ ), diisopropylamine (0.405 $\mathrm{mL}, 2.89 \mathrm{mmol})$, lactone $(0.704 \mathrm{~g}, 2.89 \mathrm{mmol})$, and $\mathrm{Me}_{3} \mathrm{SiCl}$ $(0.367 \mathrm{~mL}, 2.89 \mathrm{mmol})$ in THF $(20 \mathrm{~mL})$. Purification by chromatography on Florisil (pentane) inside a glove box afforded a viscous yellow oil $(0.270 \mathrm{~g}, 30 \%)$.

${ }^{1} \mathrm{H}$ NMR $\left(300 \mathrm{MHz}, \mathrm{C}_{6} \mathrm{D}_{6}\right) \delta$ 8.37-8.42 (m, 1H), 7.32-7.41 (m, 2H), 7.12-7.18 (m, 1H), $6.92(\mathrm{~s}, 1 \mathrm{H}), 3.09(\mathrm{~s}, 3 \mathrm{H}), 2.97(\mathrm{~s}, 2 \mathrm{H}), 1.37(\mathrm{~s}, 6 \mathrm{H}), 0.36(\mathrm{~s}, 9 \mathrm{H}) .{ }^{13} \mathrm{C} \mathrm{NMR}\left(75 \mathrm{MHz}, \mathrm{C}_{6} \mathrm{D}_{6}\right)$ $\delta$ 150.7, 137.9, 127.8, 124.6, 123.0, 122.1, 119.1, 111.9, 109.7, 81.5, 78.4, 45.7, 32.3, 29.1, 1.0. FTIR $\left(\mathrm{CH}_{2} \mathrm{Cl}_{2}\right) 2970,2880,1691,1680,1547,1534,1479,1369,1320,1173,1062 \mathrm{~cm}^{-1}$. HRMS (EI, $m / z)$ calcd. for $\mathrm{C}_{18} \mathrm{H}_{25} \mathrm{NO}_{2} \mathrm{Si}(\mathrm{M}+\mathrm{H})^{+} 316.1727$, found 316.1738 . 


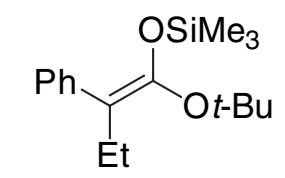

$\sim 2: 1$ mixture of isomers

A solution of $n$-BuLi (4.41 mL, $11.3 \mathrm{mmol}$ in hexanes) was added via syringe to a -78 ${ }^{\circ} \mathrm{C}$ solution of diisopropylamine $(1.58 \mathrm{~mL}, 11.3 \mathrm{mmol})$ in THF $(20 \mathrm{~mL})$. The mixture was stirred at $-78{ }^{\circ} \mathrm{C}$ for 45 minutes, and then a solution of the ester $(2.50 \mathrm{~g}, 11.3 \mathrm{mmol})$ in THF $(20 \mathrm{~mL})$ was added via cannula. The mixture was stirred at $-78^{\circ} \mathrm{C}$ for 90 minutes, and then $\mathrm{Me}_{3} \mathrm{SiCl}(1.43 \mathrm{~mL}, 11.3 \mathrm{mmol})$ was added, resulting in a clear, light-yellow solution which was warmed to room temperature and stirred for 3 hours. The THF was then removed, and the residue was taken up in pentane and filtered. The solvent was removed, and the yellow liquid was purified by fractional distillation $\left(88-91^{\circ} \mathrm{C}, 1.0\right.$ $\mathrm{mm} \mathrm{Hg})$ to afford a clear, colorless oil $(1.80 \mathrm{~g}, 55 \%)$.

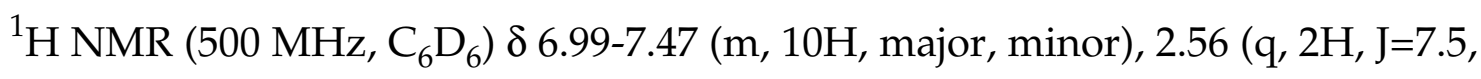
major), 2.52 (q, 2H, J=7.5, minor), 1.36 (s, 9H, major), $1.04(\mathrm{~s}, 9 \mathrm{H}$, minor), $1.03(\mathrm{t}, 3 \mathrm{H}$, $\mathrm{J}=7.2$, minor), 0.99 ( $\mathrm{t}, 3 \mathrm{H}, \mathrm{J}=7.5$, major), 0.24 ( $\mathrm{s}, 9 \mathrm{H}$, minor), -0.92 ( $\mathrm{s}, 9 \mathrm{H}$, major). ${ }^{13} \mathrm{C}$ NMR (125 MHz, $\left.\mathrm{CDCl}_{3}\right) \delta$ 149.2, 149.1, 140.9, 140.3, 130.4, 130.3, 126.1, 125.9, 109.7, 108.7, 80.5, 79.0, 55.7, 55.2, 29.8, 29.5, 24.6 (isochronous with other isomer), 14.0 (isochronous with other isomer), 1.0, 0.6. FTIR $\left(\mathrm{CH}_{2} \mathrm{Cl}_{2}\right) 2967,2876,1658,1642,1598,1367,1218$, $1148,1093,987 \mathrm{~cm}^{-1}$. 


\section{Enantioselectivity as a Function of Catalyst (eq 2)}

General. Although several of the silyl ketene acetals were purified by chromatography on Florisil inside a glove box (Section III), this level of purity is not essential for catalytic enantioselective C-acylations-we have used unpurified silyl ketene acetals, and they furnish the same ee. However, we routinely purify the silyl ketene acetals in order to accurately determine the yields of the acylation reactions.

Unless otherwise specified, all reactions are the average of two runs. These acylations were set up in a glove box, due to the moisture sensitivity of the trimethylsilyl ketene acetals, which results in lower yields when reactions are set up without a glove box.

Catalyst (-)-1. A solution of (-)-1 (2.7 mg, $0.0077 \mathrm{mmol})$ in $1.4 \mathrm{~mL}$ of $\mathrm{Et}_{2} \mathrm{O} / \mathrm{CH}_{2} \mathrm{Cl}_{2}$ (14:1) was added to a 20-mL vial containing the silyl ketene acetal $(28 \mathrm{mg}, 0.11 \mathrm{mmol})$. The pink mixture was stirred for 5 minutes at room temperature, after which $\mathrm{Ac}_{2} \mathrm{O}$ $(0.013 \mathrm{~mL}, 0.14 \mathrm{mmol})$ was added, resulting in a bright-gold solution. The vial was capped and removed from the glove box. After stirring for 24 hours at room temperature, the reaction mixture was purified directly by flash chromatography $(25 \%$ $\mathrm{Et}_{2} \mathrm{O} / 75 \%$ pentane). The product was analyzed by chiral GC (Chiraldex G-TA, $20 \mathrm{mx}$ $0.25 \mathrm{~mm}, 110{ }^{\circ} \mathrm{C}, 1.0 \mathrm{~mL} / \mathrm{min}$, retention times of enantiomers: $40.2 \mathrm{~min}$ (minor), 41.9 $\min$ (major)), which showed $42 \%$ ee.

Run 2: silyl ketene acetal (47 mg, $0.18 \mathrm{mmol}),(-)-\mathbf{1}(3.2 \mathrm{mg}, 0.0091 \mathrm{mmol}), \mathrm{Ac}_{2} \mathrm{O}$ (0.022 mL, $0.23 \mathrm{mmol})$, and $2.4 \mathrm{~mL}$ of $\mathrm{Et}_{2} \mathrm{O} / \mathrm{CH}_{2} \mathrm{Cl}_{2}$ (14:1); 37\% ee.

Catalyst (-)-2. A solution of (-)-2 (2.6 mg, $0.0069 \mathrm{mmol})$ in $1.8 \mathrm{~mL}$ of $\mathrm{Et}_{2} \mathrm{O} / \mathrm{CH}_{2} \mathrm{Cl}_{2}$ (14:1) was added to a $20-\mathrm{mL}$ vial containing the silyl ketene acetal (36 mg, $0.14 \mathrm{mmol}$ ). The pink mixture was stirred for 5 minutes at room temperature, after which $\mathrm{Ac}_{2} \mathrm{O}$ $(0.017 \mathrm{~mL}, 0.18 \mathrm{mmol})$ was added, resulting in a bright-gold solution. The vial was capped and removed from the glove box. After stirring for 24 hours at room temperature, the reaction mixture was purified directly by flash chromatography $(25 \%$ $\mathrm{Et}_{2} \mathrm{O} / 75 \%$ pentane). The product was analyzed by chiral GC, which showed $31 \%$ ee. 
Run 2: silyl ketene acetal (42 mg, $0.16 \mathrm{mmol}),(-)-2(3.0 \mathrm{mg}, 0.0080 \mathrm{mmol}), \mathrm{Ac}_{2} \mathrm{O}$ (0.019 mL, $0.21 \mathrm{mmol})$, and $2.1 \mathrm{~mL}$ of $\mathrm{Et}_{2} \mathrm{O} / \mathrm{CH}_{2} \mathrm{Cl}_{2}$ (14:1); $34 \%$ ee.

Catalyst (-)-3. A solution of (-)-3 (4.2 mg, $0.0064 \mathrm{mmol})$ in $1.7 \mathrm{~mL}$ of $\mathrm{Et}_{2} \mathrm{O} / \mathrm{CH}_{2} \mathrm{Cl}_{2}$ (14:1) was added to a $20-\mathrm{mL}$ vial containing the silyl ketene acetal (33 mg, $0.13 \mathrm{mmol}$ ). The pink mixture was stirred for 5 minutes at room temperature, after which $\mathrm{Ac}_{2} \mathrm{O}$ ( $0.016 \mathrm{~mL}, 0.17 \mathrm{mmol})$ was added. The vial was capped and removed from the glove box. After stirring for 24 hours at room temperature, the reaction mixture was purified directly by flash chromatography $\left(25 \% \mathrm{Et}_{2} \mathrm{O} / 75 \%\right.$ pentane$)$. The product was analyzed by chiral GC, which showed $87 \%$ ee.

Run 2: silyl ketene acetal (32 mg, $0.12 \mathrm{mmol}),(-)-3$ (4.0 mg, $0.0061 \mathrm{mmol}), \mathrm{Ac}_{2} \mathrm{O}$ (0.015 mL, $0.16 \mathrm{mmol})$, and $1.6 \mathrm{~mL}$ of $\mathrm{Et}_{2} \mathrm{O} / \mathrm{CH}_{2} \mathrm{Cl}_{2}(14: 1) ; 87 \%$ ee.

Catalyst (-)-4. The experimental procedure and the characterization data are located in Section V, Table 1, entry 1. 


\section{Catalytic Enantioselective Intermolecular C-Acylation of Silyl Ketene Acetals (Table 1 and eq 3)}

General. Although several of the silyl ketene acetals were purified by chromatography on Florisil inside a glove box (Section III), this level of purity is not essential for catalytic enantioselective C-acylations-we have used unpurified silyl ketene acetals, and they furnish the same ee. However, we routinely purify the silyl ketene acetals in order to accurately determine the yields of the acylation reactions.

Unless otherwise specified, all reactions are the average of two runs (one run with each enantiomer of the catalyst). The acylations were set up in a glove box (exceptions: Table 1, entries 2 and 5), due to the moisture sensitivity of the trimethylsilyl ketene acetals, which results in lower yields when reactions are set up without a glove box.

General Procedure. A solution of catalyst (0.05 equiv) in $\mathrm{Et}_{2} \mathrm{O} / \mathrm{CH}_{2} \mathrm{Cl}_{2}$ (14:1) was added to a $20-\mathrm{mL}$ vial containing the silyl ketene acetal (1.0 equiv). The resulting solution was stirred for 5 minutes at room temperature, after which $\mathrm{Ac}_{2} \mathrm{O}$ (1.3 equiv) was added. The vial was capped and removed from the glove box, and the reaction mixture was stirred for 24 hours at room temperature. The product was then purified directly by flash chromatography $\left(25 \% \mathrm{Et}_{2} \mathrm{O} / 75 \%\right.$ pentane). The catalyst was recovered by eluting with 3 volumes of $\mathrm{CH}_{2} \mathrm{Cl}_{2}$, followed by $10 \% \mathrm{Et}_{3} \mathrm{~N} / 90 \% \mathrm{EtOAc}$. The ee of the product was determined either by chiral HPLC or by chiral GC.

Table 1, entry 1. The general procedure was followed, using silyl ketene acetal (0.164 g, $0.626 \mathrm{mmol}), \mathrm{Ac}_{2} \mathrm{O}(0.0768 \mathrm{~mL}, 0.814 \mathrm{mmol}),(+)-4(0.0215 \mathrm{~g}, 0.0313 \mathrm{mmol})$, and $8.2 \mathrm{~mL}$ of $\mathrm{Et}_{2} \mathrm{O} / \mathrm{CH}_{2} \mathrm{Cl}_{2}$ (14:1) to produce $78 \%(0.113 \mathrm{~g}, 0.487 \mathrm{mmol})$ of a white crystalline solid, which was shown by chiral GC (Chiraldex G-TA, $20 \mathrm{~m} \times 0.25 \mathrm{~mm}, 110$ ${ }^{\circ} \mathrm{C}, 1.0 \mathrm{~mL} / \mathrm{min}$, retention times of enantiomers: $42.0 \mathrm{~min}$ (major), $41.3 \mathrm{~min}$ (minor)) to have $90 \%$ ee.

For run 2, the silyl ketene acetal $(0.156 \mathrm{~g}, 0.582 \mathrm{mmol}), \mathrm{Ac}_{2} \mathrm{O}(0.0714 \mathrm{~mL}, 0.757$ $\mathrm{mmol})$, and (-)-4 (0.0200 g, $0.0290 \mathrm{mmol})$ in $7.7 \mathrm{~mL}$ of $\mathrm{Et}_{2} \mathrm{O} / \mathrm{CH}_{2} \mathrm{Cl}_{2}(14: 1)$ furnished 
$81 \%$ of the product $(0.109 \mathrm{~g}, 0.470 \mathrm{mmol})$, which was shown by chiral GC to have $90 \%$ ee.

${ }^{1} \mathrm{H}$ NMR (300 MHz, $\left.\mathrm{C}_{6} \mathrm{D}_{6}\right)$ 反 7.30-7.34 (m, 2H), 6.92-7.04 (m, 3H), $3.25(\mathrm{~d}, 1 \mathrm{H}, \mathrm{J}=13.5)$, $2.10(\mathrm{~s}, 3 \mathrm{H}), 1.82(\mathrm{~d}, 1 \mathrm{H}, \mathrm{J}=13.5), 1.04(\mathrm{~s}, 3 \mathrm{H}), 0.81(\mathrm{~s}, 3 \mathrm{H}) .{ }^{13} \mathrm{C} \mathrm{NMR}\left(125 \mathrm{MHz}, \mathrm{CDCl}_{3}\right) \delta$ $201.4,172.8,138.9,129.6,128.3,126.7,82.6,68.6,44.7,29.1,28.9$, 26.5. FTIR $\left(\mathrm{CH}_{2} \mathrm{Cl}_{2}\right)$ 2983, 2932, 1750, 1718, 1559, 1455, 1373, 1275, 1182, 1151, $1097 \mathrm{~cm}^{-1}$. HRMS (ESI, $m / z$ ) calcd. for $\mathrm{C}_{14} \mathrm{H}_{16} \mathrm{O}_{3}(\mathrm{M}+\mathrm{Na})^{+}$255.0992, found 255.0993. $[\alpha]^{20} \mathrm{D}=-213^{\circ}\left(\mathrm{c}=0.89, \mathrm{CH}_{2} \mathrm{Cl}_{2}\right.$; for product with $90 \%$ ee). $\mathrm{mp} 64-66^{\circ} \mathrm{C}$.

Table 1, entry 2. The general procedure was followed, without the use of a glove box, with silyl ketene acetal (0.148 g, $0.506 \mathrm{mmol}), \mathrm{Ac}_{2} \mathrm{O}(0.0621 \mathrm{~mL}, 0.658 \mathrm{mmol}),(+)-4$ $(0.0174 \mathrm{~g}, 0.0253 \mathrm{mmol})$, and $6.7 \mathrm{~mL}$ of $\mathrm{Et}_{2} \mathrm{O} / \mathrm{CH}_{2} \mathrm{Cl}_{2}(14: 1)$ to produce $76 \%(0.101 \mathrm{~g}$, $0.385 \mathrm{mmol}$ ) of a colorless crystalline solid, which was shown by chiral GC (Chiraldex G-TA, $20 \mathrm{~m} \times 0.25 \mathrm{~mm}, 120{ }^{\circ} \mathrm{C}, 1.0 \mathrm{~mL} / \mathrm{min}$, retention times of enantiomers: $105 \mathrm{~min}$ (major), $108 \mathrm{~min}$ (minor)) to have $95 \%$ ee.

For run 2 (inside a glove box), the silyl ketene acetal $(0.150 \mathrm{~g}, 0.513 \mathrm{mmol}), \mathrm{Ac}_{2} \mathrm{O}$ $(0.0629 \mathrm{~mL}, 0.667 \mathrm{mmol})$, and $(-)-4(0.0176 \mathrm{~g}, 0.0260 \mathrm{mmol})$ in $6.8 \mathrm{~mL}$ of $\mathrm{Et}_{2} \mathrm{O} / \mathrm{CH}_{2} \mathrm{Cl}_{2}$ (14:1) furnished $80 \%$ of the product $(0.107 \mathrm{~g}, 0.408 \mathrm{mmol})$, which was shown by chiral GC to have $95 \%$ ee.

${ }^{1} \mathrm{H}$ NMR (500 MHz, $\left.\mathrm{C}_{6} \mathrm{D}_{6}\right) \delta 7.23(\mathrm{~d}, 2 \mathrm{H}, \mathrm{J}=9.0), 6.61(\mathrm{~d}, 2 \mathrm{H}, \mathrm{J}=9.0), 3.21(\mathrm{~d}, 1 \mathrm{H}$, $\mathrm{J}=13.5), 3.17(\mathrm{~s}, 3 \mathrm{H}), 2.12(\mathrm{~s}, 3 \mathrm{H}), 1.83(\mathrm{~d}, 1 \mathrm{H}, \mathrm{J}=13.5), 1.04(\mathrm{~s}, 3 \mathrm{H}), 0.82(\mathrm{~s}, 3 \mathrm{H}) .{ }^{13} \mathrm{C}$ NMR $\left(125 \mathrm{MHz}, \mathrm{CDCl}_{3}\right) \delta$ 201.1, 173.0, 160.0, 131.8, 128.5, 115.2, 81.8, 68.2, 55.1, 45.0, 29.0, 28.8, 26.2. FTIR $\left(\mathrm{CH}_{2} \mathrm{Cl}_{2}\right) 2979,2937,2839,1751,1716,1609,1512,1457,1375,1356,1254,1183$, $1031 \mathrm{~cm}^{-1}$. HRMS (EI, $m / z$ ) calcd. for $\mathrm{C}_{15} \mathrm{H}_{18} \mathrm{O}_{4}\left(\mathrm{M}^{+}\right)$262.1200, found 262.1196. $[\alpha]^{20} \mathrm{D}=$ $-235^{\circ}$ ( $\mathrm{c}=1.0, \mathrm{CH}_{2} \mathrm{Cl}_{2}$; for product with $95 \%$ ee). mp $47-49^{\circ} \mathrm{C}$.

Table 1, entry 3. The general procedure was followed, using silyl ketene acetal (0.141 g, $0.466 \mathrm{mmol}), \mathrm{Ac}_{2} \mathrm{O}(0.0572 \mathrm{~mL}, 0.606 \mathrm{mmol}),(+)-4(0.0160 \mathrm{~g}, 0.0233 \mathrm{mmol})$, and $6.1 \mathrm{~mL}$ of $\mathrm{Et}_{2} \mathrm{O} / \mathrm{CH}_{2} \mathrm{Cl}_{2}$ (14:1) to produce $85 \%$ (0.108 g, $\left.0.394 \mathrm{mmol}\right)$ of a clear, colorless oil, which was shown by chiral GC (Chiraldex G-TA, $20 \mathrm{~m} \times 0.25 \mathrm{~mm}, 150{ }^{\circ} \mathrm{C}, 1.0$ 
$\mathrm{mL} / \mathrm{min}$, retention times of enantiomers: $9.48 \mathrm{~min}$ (major), $12.9 \mathrm{~min}$ (minor)) to have $90 \%$ ee.

For run 2, the silyl ketene acetal $(0.150 \mathrm{~g}, 0.496 \mathrm{mmol}), \mathrm{Ac}_{2} \mathrm{O}(0.0609 \mathrm{~mL}, 0.645$ $\mathrm{mmol})$, and (-)-4 (0.0170 g, $0.0248 \mathrm{mmol})$ in $6.5 \mathrm{~mL}$ of $\mathrm{Et}_{2} \mathrm{O} / \mathrm{CH}_{2} \mathrm{Cl}_{2}(14: 1)$ furnished $83 \%$ of the product $(0.111 \mathrm{~g}, 0.408 \mathrm{mmol})$, which was shown by chiral GC to have $90 \%$ ee.

${ }^{1} \mathrm{H} \mathrm{NMR}\left(300 \mathrm{MHz} \mathrm{CDCl}_{3}\right) \delta 7.67(\mathrm{dd}, 2 \mathrm{H}, \mathrm{J}=8.7, \mathrm{~J}=0.6), 7.56(\mathrm{dd}, 2 \mathrm{H}, \mathrm{J}=8.7, \mathrm{~J}=0.6)$, 4.22-4.36 (m, 2H), 3.35-3.44 (m, 1H), 2.39 (ddd, 1H, J=13.2, J=7.5, J=7.5), $2.22(\mathrm{~s}, 3 \mathrm{H}) .{ }^{13} \mathrm{C}$ $\operatorname{NMR}\left(125 \mathrm{MHz}, \mathrm{CDCl}_{3}\right) \delta 200.0,173.0,140.6,130.8$ (q, J=32.8), 127.4, 126.5 (q, J=4.0) $123.8(\mathrm{q}, \mathrm{J}=272.4), 65.9,65.8,33.4,26.5$. FTIR $\left(\mathrm{CH}_{2} \mathrm{Cl}_{2}\right) 3077,3001,2921,1771,1717,1617$, $1414,1360,1328,1328,1120,1069,1019,843 \mathrm{~cm}^{-1}$. HRMS (ESI, $\left.m / z\right)$ calcd. for $\mathrm{C}_{13} \mathrm{H}_{11} \mathrm{~F}_{3} \mathrm{O}_{3}(\mathrm{M}+\mathrm{Na})^{+} 295.0552$, found 295.0555. $[\alpha]^{20} \mathrm{D}=-150^{\circ}\left(\mathrm{c}=0.32, \mathrm{CH}_{2} \mathrm{Cl}_{2}\right.$; for product with $90 \%$ ee).

Table 1, entry 4. The general procedure was followed, using silyl ketene acetal (0.150 g, $0.543 \mathrm{mmol}), \mathrm{Ac}_{2} \mathrm{O}(0.0666 \mathrm{~mL}, 0.706 \mathrm{mmol}),(-)-4(0.0186 \mathrm{~g}, 0.0270 \mathrm{mmol})$, and $7.1 \mathrm{~mL}$ of $\mathrm{Et}_{2} \mathrm{O} / \mathrm{CH}_{2} \mathrm{Cl}_{2}$ (14:1), except that the reaction was stirred for 36 hours at room temperature. The reaction produced $87 \%(0.117 \mathrm{~g}, 0.475 \mathrm{mmol})$ of a white crystalline solid, which was shown by chiral HPLC (Daicel CHIRALPAK AD, $4.6 \mathrm{~mm} \times 25 \mathrm{~cm}$, hexane/isopropanol 90:10, $1.0 \mathrm{~mL} / \mathrm{min}$, retention times of enantiomers: $4.51 \mathrm{~min}$ (minor), $5.10 \mathrm{~min}$ (major)) to have $94 \%$ ee.

For run 2, the silyl ketene acetal $(0.155 \mathrm{~g}, 0.562 \mathrm{mmol}), \mathrm{Ac}_{2} \mathrm{O}(0.0689 \mathrm{~mL}, 0.731$ $\mathrm{mmol})$, and (+)-4 (0.0193 $\mathrm{g}, 0.0281 \mathrm{mmol})$ in $7.4 \mathrm{~mL}$ of $\mathrm{Et}_{2} \mathrm{O} / \mathrm{CH}_{2} \mathrm{Cl}_{2}(14: 1)$ furnished $90 \%$ of the product $(0.125 \mathrm{~g}, 0.509 \mathrm{mmol})$, which was shown by chiral HPLC to have $96 \%$ ee.

${ }^{1} \mathrm{H}$ NMR $\left(300 \mathrm{MHz}, \mathrm{CDCl}_{3}\right) \delta$ 7.46-7.49 (m, 1H), 7.16-7.28 (m, 3H), $3.56(\mathrm{~d}, 1 \mathrm{H}$, $\mathrm{J}=13.2), 2.21(\mathrm{~s}, 3 \mathrm{H}), 2.10(\mathrm{~d}, 1 \mathrm{H}, \mathrm{J}=13.2), 2.09(\mathrm{~s}, 3 \mathrm{H}), 1.51(\mathrm{~s}, 3 \mathrm{H}), 1.27(\mathrm{~s}, 3 \mathrm{H}) .{ }^{13} \mathrm{C}$ NMR $\left(125 \mathrm{MHz}, \mathrm{CDCl}_{3}\right) \delta 203.1,173.0,138.6,135.4,132.7,128.4,127.9,127.0,83.0,69.5,43.5$, 29.5, 29.4, 27.2, 20.2. FTIR $\left(\mathrm{CH}_{2} \mathrm{Cl}_{2}\right) 3065,2978,2934,2875,1750,1716,1456,1375,1271$, $1182,1143,1104,1036,961 \mathrm{~cm}^{-1}$. HRMS (ESI, $\left.\mathrm{m} / \mathrm{z}\right)$ calcd. for $\mathrm{C}_{15} \mathrm{H}_{18} \mathrm{O}_{3}(\mathrm{M}+\mathrm{Na})^{+}$ 
269.1148, found 269.1141. $[\alpha]^{20} \mathrm{D}=+256^{\circ}\left(\mathrm{c}=0.39, \mathrm{CH}_{2} \mathrm{Cl}_{2}\right.$; for product with $94 \%$ ee). $\mathrm{mp} 67-68^{\circ} \mathrm{C}$.

Table 1, entry 5. The general procedure was followed, using silyl ketene acetal (0.150 g, $0.480 \mathrm{mmol}), \mathrm{Ac}_{2} \mathrm{O}$ (0.0589 mL, $\left.0.624 \mathrm{mmol}\right),(-)-4(0.0165 \mathrm{~g}, 0.0240 \mathrm{mmol})$, and $6.3 \mathrm{~mL}$ of $\mathrm{Et}_{2} \mathrm{O} / \mathrm{CH}_{2} \mathrm{Cl}_{2}$ (14:1) to produce $79 \%(0.107 \mathrm{~g}, 0.379 \mathrm{mmol})$ of a white crystalline solid, which was shown by chiral HPLC (Daicel CHIRALCEL OD, $4.6 \mathrm{~mm} x$ $25 \mathrm{~cm}$, hexane/isopropanol 99:1,1.0 $\mathrm{mL} / \mathrm{min}$, retention times of enantiomers: $8.68 \mathrm{~min}$ (minor), $9.76 \mathrm{~min}$ (major)) to have $99 \%$ ee.

For run 2 (outside a glove box), the silyl ketene acetal (0.155 g, $0.495 \mathrm{mmol}), \mathrm{Ac}_{2} \mathrm{O}$ $(0.0607 \mathrm{~mL}, 0.644 \mathrm{mmol})$, and (+)-4 $(0.0170 \mathrm{~g}, 0.0248 \mathrm{mmol})$ in $6.5 \mathrm{~mL}$ of $\mathrm{Et}_{2} \mathrm{O} / \mathrm{CH}_{2} \mathrm{Cl}_{2}$ (14:1) furnished $84 \%$ of the product $(0.117 \mathrm{~g}, 0.415 \mathrm{mmol})$, which was shown by chiral HPLC to have $99 \%$ ee.

${ }^{1} \mathrm{H}$ NMR $\left(300 \mathrm{MHz}, \mathrm{CDCl}_{3}\right)$ $\delta$ 7.86-7.93 (m, 2H), $7.75(\mathrm{dd}, 1 \mathrm{H}, \mathrm{J}=6.3, \mathrm{~J}=0.9)$, 7.39-7.55 $(\mathrm{m}, 4 \mathrm{H}), 3.80(\mathrm{~d}, 1 \mathrm{H}, \mathrm{J}=13.2), 2.35(\mathrm{~d}, 1 \mathrm{H}, \mathrm{J}=13.2), 2.10(\mathrm{~s}, 3 \mathrm{H}), 1.57(\mathrm{~s}, 3 \mathrm{H}), 1.20(\mathrm{~s}, 3 \mathrm{H})$. ${ }^{13} \mathrm{C}$ NMR $\left(125 \mathrm{MHz}, \mathrm{CDCl}_{3}\right) \delta 204.3,173.1,135.9,134.9,130.3,129.9,129.5,127.4,126.32$, 126.31, 125.7, 123.5, 83.4, 69.3, 44.2, 29.5, 29.4, 27.4. FTIR $\left(\mathrm{CH}_{2} \mathrm{Cl}_{2}\right) 3061,3001,2981,1747$, 1715, 1559, 1456, 1388, 1351, 1272, 1179, 1149, 1132, 1064, $953 \mathrm{~cm}^{-1}$. HRMS (EI, $\mathrm{m} / \mathrm{z}$ ) calcd. for $\mathrm{C}_{18} \mathrm{H}_{18} \mathrm{O}_{3}\left(\mathrm{M}^{+}\right)$282.1250, found 282.1259. $[\alpha]^{20} \mathrm{D}=+349^{\circ}\left(\mathrm{c}=0.64, \mathrm{CH}_{2} \mathrm{Cl}_{2}\right.$; for product with $99 \%$ ee). mp $123-126^{\circ} \mathrm{C}$.

Table 1, entry 6. The general procedure was followed, using silyl ketene acetal (0.140 g, $0.521 \mathrm{mmol}), \mathrm{Ac}_{2} \mathrm{O}(0.0639 \mathrm{~mL}, 0.678 \mathrm{mmol}),(+)-4(0.0179 \mathrm{~g}, 0.0261 \mathrm{mmol})$, and $6.9 \mathrm{~mL}$ of $\mathrm{Et}_{2} \mathrm{O} / \mathrm{CH}_{2} \mathrm{Cl}_{2}(14: 1)$ to produce $86 \%(0.108 \mathrm{~g}, 0.453 \mathrm{mmol})$ of a clear, colorless oil, which was shown by chiral HPLC (Daicel CHIRALCEL OD, $4.6 \mathrm{~mm} \times 25 \mathrm{~cm}$, hexane/isopropanol 99:1,1.0 $\mathrm{mL} / \mathrm{min}$, retention times of enantiomers: $8.79 \mathrm{~min}$ (major), $9.99 \mathrm{~min}$ (minor)) to have $75 \%$ ee.

For run 2, the silyl ketene acetal $(0.158 \mathrm{~g}, 0.588 \mathrm{mmol}), \mathrm{Ac}_{2} \mathrm{O}(0.0721 \mathrm{~mL}, 0.764$ $\mathrm{mmol})$, and (-)-4 (0.0202 $\mathrm{g}, 0.0290 \mathrm{mmol})$ in $7.7 \mathrm{~mL}$ of $\mathrm{Et}_{2} \mathrm{O} / \mathrm{CH}_{2} \mathrm{Cl}_{2}(14: 1)$ furnished $81 \%$ of the product $(0.113 \mathrm{~g}, 0.474 \mathrm{mmol})$, which was shown by chiral HPLC to have $77 \%$ ee. 
${ }^{1} \mathrm{H}$ NMR $\left(300 \mathrm{MHz}, \mathrm{CDCl}_{3}\right.$ ) $\delta 7.32(\mathrm{dd}, 1 \mathrm{H}, \mathrm{J}=5.1, \mathrm{~J}=1.2), 7.17$ (dd, 1H, J=3.6, J=1.2), 7.20 (dd, 1H, J=5.1, J=3.6), 3.41 (d, 1H, J=13.2), 2.41 (d, 1H, J=13.2), 2.33 (s, 3H), 1.45 (s, 3H), $1.32(\mathrm{~s}, 3 \mathrm{H}) .{ }^{13} \mathrm{C} \mathrm{NMR}\left(125 \mathrm{MHz}, \mathrm{CDCl}_{3}\right) \delta 200.1,172.4,140.6,127.5,126.7,126.4$, 83.2, 65.0, 45.1, 29.1, 28.5, 25.6. FTIR (neat) 3109, 2980, 2935, 1757, 1717, 1456, 1376, 1272, 1132, $961 \mathrm{~cm}^{-1}$. HRMS (ESI, $\left.\mathrm{m} / z\right)$ calcd. for $\mathrm{C}_{12} \mathrm{H}_{14} \mathrm{O}_{3} \mathrm{~S}(\mathrm{M}+\mathrm{Na})^{+} 261.0556$, found 261.0566. $[\alpha]^{20} \mathrm{D}=-154^{\circ}\left(\mathrm{c}=0.54, \mathrm{CH}_{2} \mathrm{Cl}_{2}\right.$; for product with $75 \%$ ee).

Table 1, entry 7. The general procedure was followed, using silyl ketene acetal (0.148 g, $0.551 \mathrm{mmol}), \mathrm{Ac}_{2} \mathrm{O}$ (0.0675 mL, $\left.0.716 \mathrm{mmol}\right),(+)-4(0.0189 \mathrm{~g}, 0.0275 \mathrm{mmol})$, and $7.2 \mathrm{~mL}$ of $\mathrm{Et}_{2} \mathrm{O} / \mathrm{CH}_{2} \mathrm{Cl}_{2}(14: 1)$ to produce $84 \%(0.110 \mathrm{~g}, 0.460 \mathrm{mmol})$ of a clear, colorless oil, which was shown by chiral HPLC (Daicel CHIRALCEL OD, $4.6 \mathrm{~mm} \times 25 \mathrm{~cm}$, hexane/isopropanol 99:1,1.0 $\mathrm{mL} / \mathrm{min}$, retention times of enantiomers: $8.99 \mathrm{~min}$ (major), $9.84 \mathrm{~min}$ (minor)) to have $86 \%$ ee.

For run 2, the silyl ketene acetal $(0.133 \mathrm{~g}, 0.495 \mathrm{mmol}), \mathrm{Ac}_{2} \mathrm{O}(0.0607 \mathrm{~mL}, 0.644$ $\mathrm{mmol})$, and (-)-4 (0.0170 g, $0.0248 \mathrm{mmol})$ in $6.5 \mathrm{~mL}$ of $\mathrm{Et}_{2} \mathrm{O} / \mathrm{CH}_{2} \mathrm{Cl}_{2}$ (14:1) furnished $88 \%$ of the product $(0.104 \mathrm{~g}, 0.436 \mathrm{mmol})$, which was shown by chiral HPLC to have $88 \%$ ee.

${ }^{1} \mathrm{H} \mathrm{NMR}\left(300 \mathrm{MHz}, \mathrm{CDCl}_{3}\right) \delta 7.46(\mathrm{dd}, 1 \mathrm{H}, \mathrm{J}=3.0, \mathrm{~J}=1.2), 7.36(\mathrm{dd}, 1 \mathrm{H}, \mathrm{J}=5.1, \mathrm{~J}=3.0)$, $6.97(\mathrm{dd}, 1 \mathrm{H}, \mathrm{J}=5.1, \mathrm{~J}=1.2), 3.34(\mathrm{~d}, 1 \mathrm{H}, \mathrm{J}=13.5), 2.31(\mathrm{~d}, 1 \mathrm{H}, \mathrm{J}=13.5), 2.25(\mathrm{~s}, 3 \mathrm{H}), 1.42(\mathrm{~s}$, $3 \mathrm{H}), 1.32(\mathrm{~s}, 3 \mathrm{H}) .{ }^{13} \mathrm{C} \mathrm{NMR}\left(125 \mathrm{MHz}, \mathrm{CDCl}_{3}\right) \delta 200.8,173.1,138.2,127.6,126.1,123.0$, 82.8, 65.4, 43.9, 29.1, 28.7, 26.0. FTIR (neat) 3109, 2980, 2935, 1752, 1717, 1456, 1375, 1272, 1180, 1131, 1096, $960 \mathrm{~cm}^{-1}$. HRMS (ESI, $\left.\mathrm{m} / z\right)$ calcd. for $\mathrm{C}_{12} \mathrm{H}_{14} \mathrm{O}_{3} \mathrm{~S}(\mathrm{M}+\mathrm{Na})+261.0556$, found 261.0565. $[\alpha]^{20} \mathrm{D}=-209^{\circ}$ ( $\mathrm{c}=0.64, \mathrm{CH}_{2} \mathrm{Cl}_{2}$; for product with $86 \%$ ee).

Table 1, entry 8 . The general procedure was followed, using silyl ketene acetal (0.163 g, $0.679 \mathrm{mmol}), \mathrm{Ac}_{2} \mathrm{O}(0.0832 \mathrm{~mL}, 0.882 \mathrm{mmol}),(-)-4(0.0233 \mathrm{~g}, 0.0340 \mathrm{mmol})$, and $8.9 \mathrm{~mL}$ of $\mathrm{Et}_{2} \mathrm{O} / \mathrm{CH}_{2} \mathrm{Cl}_{2}$ (14:1) to produce $70 \%(0.100 \mathrm{~g}, 0.476 \mathrm{mmol})$ of a clear, colorless oil, which was shown by chiral HPLC (Daicel CHIRALPAK AD, $4.6 \mathrm{~mm} \times 25 \mathrm{~cm}$, hexane/isopropanol 90:10, $1.0 \mathrm{~mL} / \mathrm{min}$, retention times of enantiomers: $7.25 \mathrm{~min}$ (minor), $7.91 \mathrm{~min}$ (major)) to have $80 \%$ ee. 
For run 2, the silyl ketene acetal $(0.162 \mathrm{~g}, 0.673 \mathrm{mmol}), \mathrm{Ac}_{2} \mathrm{O}(0.0825 \mathrm{~mL}, 0.875$ mmol), and (+)-4 (0.0231 g, $0.0336 \mathrm{mmol})$ in $8.9 \mathrm{~mL}$ of $\mathrm{Et}_{2} \mathrm{O} / \mathrm{CH}_{2} \mathrm{Cl}_{2}(14: 1)$ furnished $76 \%$ of the product $(0.108 \mathrm{~g}, 0.514 \mathrm{mmol})$, which was shown by chiral HPLC to have $80 \%$ ee.

${ }^{1} \mathrm{H}$ NMR $\left(300 \mathrm{MHz} \mathrm{CDCl}_{3}\right) \delta 7.50(\mathrm{dd}, 1 \mathrm{H}, \mathrm{J}=2.7, \mathrm{~J}=2.4), 7.39(\mathrm{dd}, 1 \mathrm{H}, \mathrm{J}=5.1, \mathrm{~J}=2.7)$, $7.04(\mathrm{dd}, 1 \mathrm{H}, \mathrm{J}=5.1, \mathrm{~J}=2.4), 4.22-4.35(\mathrm{~m}, 2 \mathrm{H}), 3.27-3.35(\mathrm{~m}, 1 \mathrm{H}), 2.42(\mathrm{~d}$ app t, 1H, J=12.9, $\mathrm{J}=7.5), 2.27$ (s, 3H). ${ }^{13} \mathrm{C}$ NMR $\left(125 \mathrm{MHz}, \mathrm{CDCl}_{3}\right) \delta 201.3,174.3,137.0,128.1,126.7,124.0$, 66.7, 63.4, 33.3, 26.7. FTIR (neat) 3108, 2978, 2917, 1771, 1716, 1456, 1419, 1373, $1162 \mathrm{~cm}^{-1}$. HRMS (ESI, $m / z$ ) calcd. for $\mathrm{C}_{10} \mathrm{H}_{10} \mathrm{O}_{3} \mathrm{~S}(\mathrm{M}+\mathrm{Na})^{+} 233.0243$, found 233.0244. $[\alpha]^{20} \mathrm{D}=+174^{\circ}$ (c $=0.23, \mathrm{CH}_{2} \mathrm{Cl}_{2}$; for product with $80 \%$ ee).

Table 1, entry 9. The general procedure was followed, using silyl ketene acetal (0.163 g, $0.518 \mathrm{mmol}), \mathrm{Ac}_{2} \mathrm{O}(0.0636 \mathrm{~mL}, 0.674 \mathrm{mmol}),(-)-4(0.0178 \mathrm{~g}, 0.0260 \mathrm{mmol})$, and $6.8 \mathrm{~mL}$ of $\mathrm{Et}_{2} \mathrm{O} / \mathrm{CH}_{2} \mathrm{Cl}_{2}$ (14:1) to produce $94 \%(0.139 \mathrm{~g}, 0.489 \mathrm{mmol})$ of a white crystalline solid, which was shown by chiral HPLC (Daicel CHIRALCEL OD, $4.6 \mathrm{~mm} x$ $25 \mathrm{~cm}$, hexane/isopropanol 90:10,1.0 mL/min, retention times of enantiomers: $6.10 \mathrm{~min}$ (minor), $7.20 \mathrm{~min}$ (major)) to have $95 \%$ ee.

For run 2, the silyl ketene acetal $(0.139 \mathrm{~g}, 0.440 \mathrm{mmol}), \mathrm{Ac}_{2} \mathrm{O}(0.0539 \mathrm{~mL}, 0.572$ $\mathrm{mmol})$, and (+)-4 (0.0151 g, $0.0220 \mathrm{mmol})$ in $5.8 \mathrm{~mL}$ of $\mathrm{Et}_{2} \mathrm{O} / \mathrm{CH}_{2} \mathrm{Cl}_{2}(14: 1)$ furnished $87 \%$ of the product $(0.109 \mathrm{~g}, 0.383 \mathrm{mmol})$, which was shown by chiral HPLC to have $92 \%$ ee.

${ }^{1} \mathrm{H}$ NMR $\left(300 \mathrm{MHz}, \mathrm{CDCl}_{3}\right)$ \& 7.23-7.38 (m, 4H), 7.07-7.13 (m, 1H), $3.81(\mathrm{~s}, 3 \mathrm{H}), 3.61$ $(\mathrm{d}, 1 \mathrm{H}, \mathrm{J}=13.2), 2.36(\mathrm{~d}, 1 \mathrm{H}, \mathrm{J}=13.2), 2.23(\mathrm{~s}, 3 \mathrm{H}), 1.46(\mathrm{~s}, 3 \mathrm{H}), 1.33(\mathrm{~s}, 3 \mathrm{H}) .{ }^{13} \mathrm{C} \mathrm{NMR}(125$ $\left.\mathrm{MHz}_{,} \mathrm{CDCl}_{3}\right) \delta$ 201.9, 173.7, 137.6, 127.6, 125.3, 122.5, 120.2, 119.3, 112.3, 109.9, 83.3, 63.1, 42.8, 33.1, 29.6, 28.9, 25.7. FTIR $\left(\mathrm{CH}_{2} \mathrm{Cl}_{2}\right) 3125,3056,2978,2934,1751,1716,1540,1473$, $1457,1375,1272,1146,1071,965 \mathrm{~cm}^{-1}$. HRMS (EI, $\left.\mathrm{m} / \mathrm{z}\right)$ calcd. for $\mathrm{C}_{17} \mathrm{H}_{19} \mathrm{NO}_{3}\left(\mathrm{M}^{+}\right)$ 285.1360, found 285.1357. $[\alpha]^{20} \mathrm{D}=-348^{\circ}\left(\mathrm{c}=0.62, \mathrm{CH}_{2} \mathrm{Cl}_{2}\right.$; for product with $92 \%$ ee). $\mathrm{mp}$ $84-87^{\circ} \mathrm{C}$.

Eq 3. The silyl ketene acetal $(0.116 \mathrm{~g}, 0.397 \mathrm{mmol})$, a solution of $(+)-4(0.0545 \mathrm{~g}$, $0.0794 \mathrm{mmol})$ in $\mathrm{CH}_{2} \mathrm{Cl}_{2}(8.8 \mathrm{~mL})$, and $\mathrm{Ac}_{2} \mathrm{O}(0.0936 \mathrm{~mL}, 0.993 \mathrm{mmol})$ were added to a 
screwcap vial, and the resulting solution was stirred for 72 hours at room temperature. Workup and purification furnished $81 \%(0.0842 \mathrm{~g}, 0.321 \mathrm{mmol})$ of a clear, colorless oil, which was shown by chiral GC (Chiraldex G-TA, $20 \mathrm{~m} \times 0.25 \mathrm{~mm}, 100{ }^{\circ} \mathrm{C}, 1.0 \mathrm{~mL} / \mathrm{min}$, retention times of enantiomers: $14.0 \mathrm{~min}$ (minor), $15.2 \mathrm{~min}$ (major)) to have $91 \%$ ee.

For run 2, the silyl ketene acetal $(0.110 \mathrm{~g}, 0.375 \mathrm{mmol}), \mathrm{Ac}_{2} \mathrm{O}(0.0885 \mathrm{~mL}, 0.938$ $\mathrm{mmol})$, and (-)-4 $(0.0516 \mathrm{~g}, 0.0751 \mathrm{mmol})$ in $8.5 \mathrm{~mL}$ of $\mathrm{CH}_{2} \mathrm{Cl}_{2}$ furnished $83 \%$ of the product $(0.0814 \mathrm{~g}, 0.310 \mathrm{mmol})$, which was shown by chiral GC to have $90 \%$ ee.

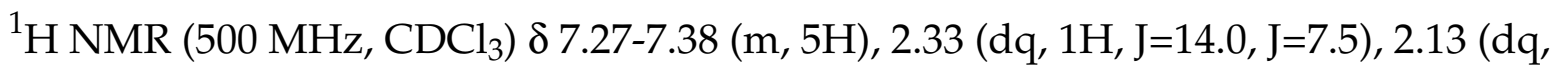
$1 \mathrm{H}, \mathrm{J}=14.0, \mathrm{~J}=7.5), 2.08$ (s, 3H), 1.51 (s, 9H), 0.86 (app t, 3H, J=7.5). ${ }^{13} \mathrm{C}$ NMR (125 MHz, $\left.\mathrm{CDCl}_{3}\right) \delta 204.0,170.2,137.4,128.7,128.3,127.6,82.6,69.7,28.5,28.1,27.9,9.5$. FTIR (thin film) $2978,1737,1713,1495,1370,1248,1156,1126,841,700 \mathrm{~cm}^{-1} .[\alpha]^{20} \mathrm{D}=-69.9^{\circ}$ (c=0.70, $\mathrm{CHCl}_{3}$; for product with $91 \%$ ee). 


\section{Mechanistic Studies (Table 2)}

Table 2, entry 1. A solution of the silyl ketene acetal $(0.020 \mathrm{~g}, 0.066 \mathrm{mmol})$ and 1,4dioxane (internal standard; $0.0011 \mathrm{~mL}, 0.013 \mathrm{mmol})$ in $\mathrm{CD}_{2} \mathrm{Cl}_{2}(0.80 \mathrm{~mL})$ was added to a screwcap NMR tube. Immediately prior to data acquisition, $\mathrm{Ac}_{2} \mathrm{O}(0.0062 \mathrm{~mL}, 0.066$ mmol) was added. The reaction was monitored by ${ }^{1} \mathrm{H}$ NMR spectroscopy after reaction times of 5 minutes, 30 minutes, 3.5 hours, 30 hours, 60 hours, and 100 hours. All of the spectra showed only unreacted starting materials.

Table 2, entry 2. A solution of the silyl ketene acetal $(0.020 \mathrm{~g}, 0.066 \mathrm{mmol})$ in $\mathrm{CD}_{2} \mathrm{Cl}_{2}(0.30 \mathrm{~mL})$ and a solution of $(-)-4(0.0023 \mathrm{mg}, 0.0033 \mathrm{mmol})$ and 1,4-dioxane (internal standard; $0.56 \mu \mathrm{L}, 0.0066 \mathrm{mmol})$ in $\mathrm{CD}_{2} \mathrm{Cl}_{2}(0.40 \mathrm{~mL})$ were added in turn to a screwcap NMR tube. Immediately prior to data acquisition, a solution of $\mathrm{Ac}_{2} \mathrm{O}(0.0063$ $\mathrm{mL}, 0.0662 \mathrm{mmol})$ in $\mathrm{CD}_{2} \mathrm{Cl}_{2}(0.10 \mathrm{~mL})$ was added. The reaction was monitored by ${ }^{1} \mathrm{H}$ NMR. Spectra were collected every 2 minutes for the first 50 minutes; the data showed a $\mathrm{t}_{1 / 2}$ of 17 minutes.

The reaction mixture was passed through a plug of silica gel $\left(\mathrm{CH}_{2} \mathrm{Cl}_{2}\right.$ as the eluent) and concentrated. The product was shown by chiral GC (Chiraldex G-TA, $20 \mathrm{~m} \times 0.25$ $\mathrm{mm}, 150{ }^{\circ} \mathrm{C}, 1.0 \mathrm{~mL} / \mathrm{min}$, retention times of enantiomers: $9.48 \mathrm{~min}$ (major), $12.9 \mathrm{~min}$ (minor)) to have $87 \%$ ee.

Table 2, entry 3. ${ }^{2}$ Inside a glove box, acetyl chloride $(0.0010 \mathrm{~mL}, 0.13 \mathrm{mmol})$ was added to a deep-purple solution of (+)-4 $(0.035 \mathrm{~g}, 0.051 \mathrm{mmol})$ in $\mathrm{CH}_{2} \mathrm{Cl}_{2}(5 \mathrm{~mL})$. After $\sim 15$ minutes, the $\mathrm{CH}_{2} \mathrm{Cl}_{2}$ and excess acetyl chloride were removed by vacuum. The resulting blue-green solid was dissolved in $\mathrm{CH}_{2} \mathrm{Cl}_{2}(2 \mathrm{~mL})$ and then evaporated to dryness (to remove residual acetyl chloride). A solution of $\mathrm{AgSbF}_{6}(0.018 \mathrm{~g}, 0.052$ mmol) in acetonitrile $(3 \mathrm{~mL})$ was added. The resulting mixture was filtered through an acrodisc to remove the $\mathrm{AgCl}$, then evaporated to provide a green residue, which was

(2) The DMAP analogue of this complex has been prepared and fully characterized, including an X-ray crystal structure: Tao, B.; Ruble, J. C.; Hoic, D. A.; Fu, G. C. J. Am. Chem. Soc. 1999, 121, 5091-5092. 
washed several times with $\mathrm{CH}_{2} \mathrm{Cl}_{2} /$ pentane (1:1) to afford a crystalline green solid $(0.049 \mathrm{~g}, 0.051 \mathrm{mmol})$ in quantitative yield.

${ }^{1} \mathrm{H}$ NMR $\left(300 \mathrm{MHz}, \mathrm{CD}_{2} \mathrm{Cl}_{2}\right) \delta 8.15(\mathrm{~d}, 1 \mathrm{H}, \mathrm{J}=8.1), 7.26(\mathrm{t}, 5 \mathrm{H}, \mathrm{J}=7.5), 7.12(\mathrm{t}, 10 \mathrm{H}$, $\mathrm{J}=7.5), 6.86(\mathrm{~d}, 10 \mathrm{H}, \mathrm{J}=7.5), 6.41(\mathrm{dd}, 1 \mathrm{H}, \mathrm{J}=3.0, \mathrm{~J}=1.2), 6.28$ (d, 1H, J=8.4), 5.10 (dd, 1H, $\mathrm{J}=3.0, \mathrm{~J}=1.2), 4.89(\mathrm{t}, 1 \mathrm{H}, \mathrm{J}=3.0), 3.63-3.90(\mathrm{~m}, 4 \mathrm{H}), 2.45(\mathrm{~s}, 3 \mathrm{H}), 2.05-2.31(\mathrm{~m}, 4 \mathrm{H}) .{ }^{13} \mathrm{C}$ $\operatorname{NMR}\left(75 \mathrm{MHz}, \mathrm{CDCl}_{3}\right) \delta 170.3,165.6,141.1,133.5,132.4,128.0,127.9,101.5,100.4,88.4$, 82.7, 71.8, 70.4, 70.2, 54.5, 53.9, 26.2, 24.8, 23.6. FTIR (KBr) 3056, 1735, 1609, 1560, 1507, 1219, 1173, 990, 702, $658 \mathrm{~cm}^{-1}$. Anal. Calcd. for $\mathrm{C}_{49} \mathrm{H}_{41} \mathrm{~F}_{6} \mathrm{FeN}_{2} \mathrm{OSb}$ (965.5): C, 60.96; $\mathrm{H}$, 4.28; N, 2.90. Found: $\mathrm{C}, 60.66 ; \mathrm{H}, 3.90 ; \mathrm{N}, 2.76 .[\alpha]^{25} \mathrm{D}=+72^{\circ}\left(\mathrm{c}=0.09, \mathrm{CH}_{2} \mathrm{Cl}_{2}\right.$; for product with $>99 \%$ ee). $\mathrm{mp} 242-244^{\circ} \mathrm{C}$.

A solution of (+)-9 (0.049 g, $0.051 \mathrm{mmol})$ and 1,4-dioxane (internal standard; 0.0011 $\mathrm{mL}, 0.013 \mathrm{mmol})$ in $\mathrm{CD}_{2} \mathrm{Cl}_{2}(0.50 \mathrm{~mL})$ was added to a screwcap NMR tube. Immediately prior to data acquisition, a solution of the silyl ketene acetal $(0.015 \mathrm{~g}, 0.051$ mmol) in $\mathrm{CD}_{2} \mathrm{Cl}_{2}(0.30 \mathrm{~mL})$ was added. The reaction was monitored by ${ }^{1} \mathrm{H} \mathrm{NMR}$ spectroscopy after reaction times of 5 minutes, 72 minutes, 10 hours, and 60 hours. All of the spectra showed only unreacted starting materials.

Table 2, entry 4. A stock solution of the silyl ketene acetal was prepared by the addition of $\mathrm{CD}_{2} \mathrm{Cl}_{2}(1.0 \mathrm{~mL})$ and 1,4-dioxane (internal standard; $\left.6.0 \mu \mathrm{L}\right)$ to the silyl ketene acetal $(42.0 \mathrm{mg}, 0.138 \mathrm{mmol})$. Another stock solution was prepared by the addition of $\mathrm{CD}_{2} \mathrm{Cl}_{2}(1 \mathrm{~mL})$ to $\mathrm{Me}_{4} \mathrm{~N}[\mathrm{OAc}](5.3 \mathrm{mg}, 0.040 \mathrm{mmol})$. To a screwcap NMR tube was added $0.35 \mathrm{~mL}$ of the stock solution of the silyl ketene acetal, then $\mathrm{CD}_{2} \mathrm{Cl}_{2}$ $(0.40 \mathrm{~mL})$, and then $62 \mu \mathrm{L}$ of the stock solution of $\mathrm{Me}_{4} \mathrm{~N}[\mathrm{OAc}]$. After $\sim 10$ minutes, neat $\mathrm{Ac}_{2} \mathrm{O}(6.1 \mu \mathrm{L}, 0.064 \mathrm{mmol})$ was added. $\mathrm{A}^{1} \mathrm{H}$ NMR spectrum after 2 minutes indicated $\sim 80 \%$ conversion to product (versus 1,4-dioxane). 


\section{Determination of the Absolute Configuration of the Product}
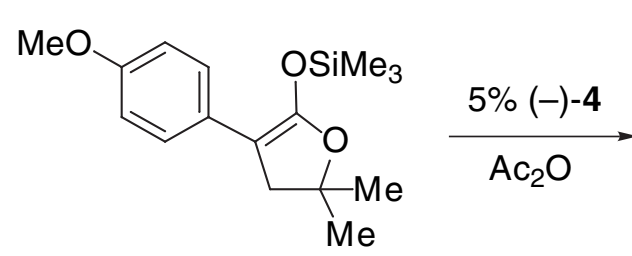

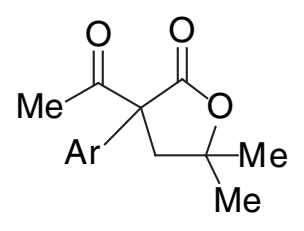

$95 \%$ ee

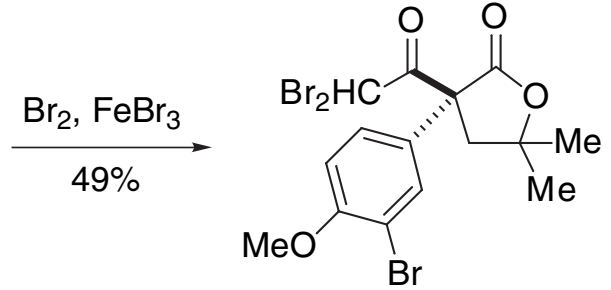

stereochemistry determined

by X-ray crystallography

$\mathrm{FeBr}_{3}(0.0100 \mathrm{~g}, 0.0330 \mathrm{mmol})$ and a solution of bromine $(0.0569 \mathrm{~mL}, 1.12 \mathrm{mmol})$ in $\mathrm{CHCl}_{3}(1.50 \mathrm{~mL})$ were added in turn to a solution of the lactone $(0.0975 \mathrm{~g}, 0.372 \mathrm{mmol})$ in $\mathrm{CHCl}_{3}(2.0 \mathrm{~mL})$. The resulting mixture was stirred at room temperature for 34 hours, after which additional $\mathrm{FeBr}_{3}(0.0150 \mathrm{~g}, 0.0507 \mathrm{mmol})$ and bromine $(0.0300 \mathrm{~mL}, 0.588$ mmol) were added. The mixture was stirred at room temperature for 30 hours, and then it was poured into saturated aqueous $\mathrm{Na}_{2} \mathrm{~S}_{2} \mathrm{O}_{3}$ and extracted with $\mathrm{Et}_{2} \mathrm{O}(3 \times 20$ $\mathrm{mL})$. The organic layer was washed with water $(1 \times 30 \mathrm{~mL})$ and dried over $\mathrm{Na}_{2} \mathrm{SO}_{4}$. The solvent was removed, and the resulting off-white solid was purified by flash chromatography (25\% $\mathrm{Et}_{2} \mathrm{O} / 75 \%$ pentane), which furnished $49 \%$ of a white crystalline solid (0.0890 g, $0.212 \mathrm{mmol})$.

${ }^{1} \mathrm{H}$ NMR (300 MHz, $\left.\mathrm{CDCl}_{3}\right) \delta 7.60(\mathrm{~d}, 1 \mathrm{H}, \mathrm{J}=2.7), 7.37(\mathrm{dd}, 1 \mathrm{H}, \mathrm{J}=9.0, \mathrm{~J}=3.0), 6.92(\mathrm{~d}$, $1 \mathrm{H}, \mathrm{J}=8.7), 6.57(\mathrm{~s}, 1 \mathrm{H}), 3.94(\mathrm{~s}, 3 \mathrm{H}), 3.43(\mathrm{~d}, 1 \mathrm{H}, \mathrm{J}=13.5), 2.36(\mathrm{~d}, 1 \mathrm{H}, \mathrm{J}=13.5), 1.50$ (s, 3H), $1.36(\mathrm{~s}, 3 \mathrm{H}) .{ }^{13} \mathrm{C}$ NMR $\left(125 \mathrm{MHz}, \mathrm{CDCl}_{3}\right) \delta 191.1,171.5,156.7,132.4,128.5,127.8,113.2$, 112.5, 83.7, 65.2, 56.6, 45.2, 36.1, 29.1, 28.7. FTIR $\left(\mathrm{CH}_{2} \mathrm{Cl}_{2}\right)$ 3029, 2982, 2943, 2904, 2483, $1749,1733,1559,1540,1497,1457,1388,1273,1199 \mathrm{~cm}^{-1}$. HRMS (ESI, m/z) calcd. for $\mathrm{C}_{15} \mathrm{H}_{15} \mathrm{Br}_{3} \mathrm{O}_{4}(\mathrm{M}+\mathrm{H})+496.8613$, found 496.8613. [ $\left.\alpha\right]^{20} \mathrm{D}=+235^{\circ}\left(\mathrm{c}=0.25, \mathrm{CH}_{2} \mathrm{Cl}_{2}\right.$; for product with $95 \%$ ee). $\operatorname{mp} 148-152{ }^{\circ} \mathrm{C}$.

X-ray quality crystals were grown from $\mathrm{Et}_{2} \mathrm{O} /$ pentane, and the configuration of the quaternary center was assigned as $(\mathrm{R})$ by single crystal X-ray diffraction. 


\section{X-ray Crystal Structure}

A colorless solution of the lactone in $\mathrm{Et}_{2} \mathrm{O}$ was prepared. Crystals suitable for $\mathrm{X}$ ray structural analysis were obtained by pentane diffusion. A clear, colorless block of dimensions $0.57 \times 0.18 \times 0.09 \mathrm{~mm}^{3}$ was mounted under STP and transferred to a Bruker AXS/CCD three-circle diffractometer ( $\chi$ fixed at $54.78^{\circ}$ ) equipped with a cold stream of $\mathrm{N}_{2}$ gas. An initial unit cell was determined by harvesting reflections $\mathrm{I}>20 \sigma(\mathrm{I})$ from 45 $x$ 10-s frames of $0.30^{\circ} \omega$ scan data with monochromated Mo $K_{\alpha}$ radiation $(\lambda=0.71073$ A). The cell thus determined was orthorhombic.

A hemisphere of data was then collected using $\omega$ scans of $0.30^{\circ}$ and 10 -s frames. The raw data frames were integrated using the Bruker program SAINT+ for NT version 6.01. Actual integration was performed with constant spot sizes of $1.6^{\circ}$ in the detector plane and $0.6^{\circ}$ in $\omega$. The data that were collected (6854 total reflections, 2473 unique, $R_{\text {int }}=0.2631$ ) had the following Miller index ranges: -7 to 7 in $h,-13$ to 12 in $k$, and -13 to 22 in 1 . The data were corrected for Lorentz and polarization effects. No absorption correction was applied.

All aspects of the solution and refinement were handled by SHELXTL NT version 5.10. ${ }^{3}$ The structure was solved by direct methods in the orthorhombic space group P2(1)2(1)2(1), $a=6.7476(8) \AA ; b=12.5932(15) \AA ; c=20.305(2) \AA ; \alpha=90^{\circ} ; \beta=90^{\circ} ; \gamma=90^{\circ}$, and refined using standard difference Fourier techniques. Final, full-matrix leastsquares refinement (2473 data for 203 parameters) on $\mathrm{F}^{2}$ yielded residuals of $\mathrm{R}_{1}$ and $\mathrm{wR}_{2}$ of 0.0878 and 0.2031 for data $\mathrm{I}>2 \sigma(\mathrm{I})$, and 0.0906 and 0.2070 , respectively, for all data. During the final refinement, all non-hydrogen atoms were treated anisotropically. Hydrogen atoms were included in calculated positions and refined isotropically on a riding model. A secondary extinction coefficient of $0.021(3)$ was used in the refinement. Residual electron density amounted to a maximum of $1.886 \mathrm{e} / \AA^{3}$ and a minimum of $-2.216 \mathrm{e} / \AA^{3}$.

(3) SHELXTL: Bruker AXS, Inc., SHELXTLTM Reference Manual Version 5.1, 1997. 
Crystallographic data (excluding structure factors) have been deposited with the Cambridge Crystallographic Data Centre as supplementary publication no. CCDC178106. Copies of the data can be obtained free of charge on application to CCDC, 12 Union Road, Cambridge CB2 1EZ, UK (fax: (+44)1223-336-033; E-mail: deposit@ccdc.cam.ac.uk).

Tables 1-6 provide the full crystallographic data for the X-ray structure. 


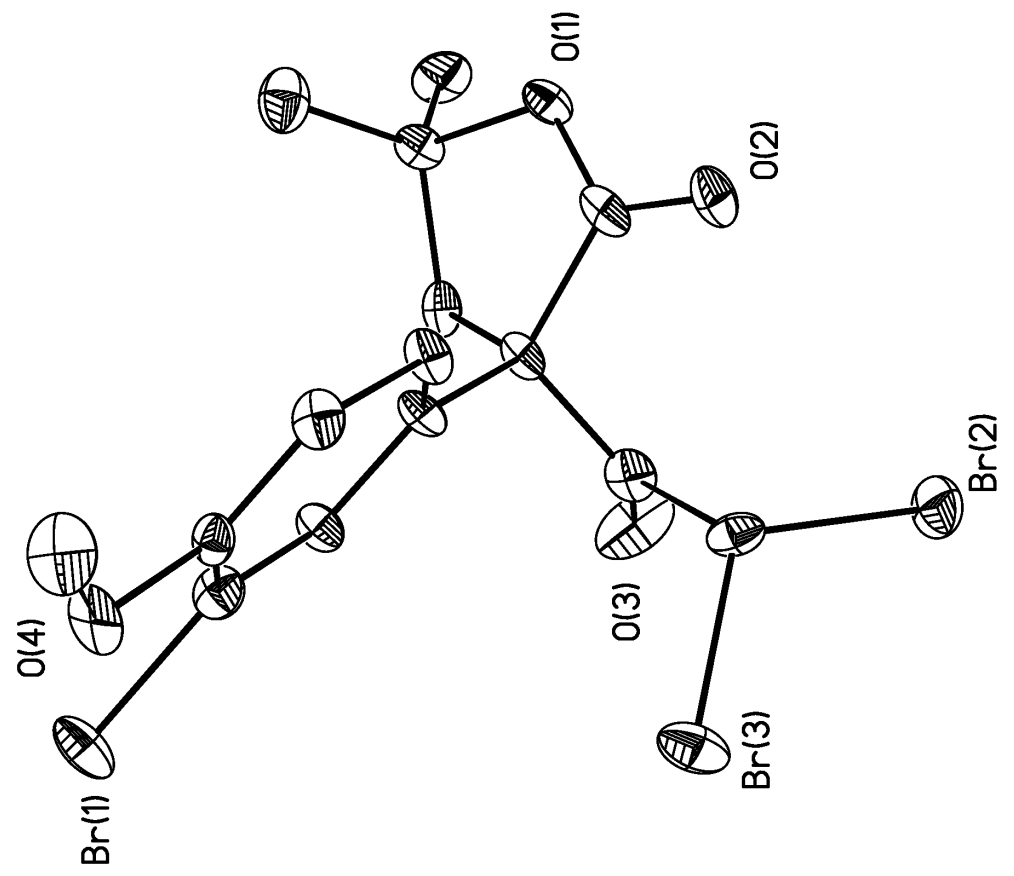


Table 1. Crystal data and structure refinement for 02015am2.

\begin{tabular}{|c|c|c|}
\hline Identification code & \multicolumn{2}{|l|}{ 02015am2 } \\
\hline Empirical formula & \multicolumn{2}{|l|}{ C15 H15 Br3 O4 } \\
\hline Formula weight & \multicolumn{2}{|l|}{499.00} \\
\hline Temperature & \multicolumn{2}{|l|}{$183(2) \mathrm{K}$} \\
\hline Wavelength & \multicolumn{2}{|l|}{$0.71073 \AA$} \\
\hline Crystal system & \multicolumn{2}{|l|}{ Orthorhombic } \\
\hline Space group & \multicolumn{2}{|l|}{$\mathrm{P} 2(1) 2(1) 2(1)$} \\
\hline \multirow[t]{3}{*}{ Unit cell dimensions } & $a=6.7476(8) \AA$ & $\alpha=90^{\circ}$ \\
\hline & $\mathrm{b}=12.5932(15) \AA$ & $\beta=90^{\circ}$ \\
\hline & $c=20.305(2) \AA$ & $\gamma=90^{\circ}$ \\
\hline Volume & \multicolumn{2}{|l|}{$1725.4(3) \AA^{3}$} \\
\hline Z & \multicolumn{2}{|c|}{4} \\
\hline Density (calculated) & \multicolumn{2}{|l|}{$1.921 \mathrm{Mg} / \mathrm{m}^{3}$} \\
\hline Absorption coefficient & \multicolumn{2}{|l|}{$7.027 \mathrm{~mm}^{-1}$} \\
\hline $\mathrm{F}(000)$ & \multicolumn{2}{|l|}{968} \\
\hline Crystal size & \multicolumn{2}{|c|}{$0.09 \times 0.18 \times 0.57 \mathrm{~mm}^{3}$} \\
\hline Theta range for data collection & \multicolumn{2}{|l|}{3.18 to $23.29^{\circ}$. } \\
\hline Index ranges & \multicolumn{2}{|c|}{$-7<=\mathrm{h}<=7,-13<=\mathrm{k}<=12,-13<=\mathrm{l}<=22$} \\
\hline Reflections collected & \multicolumn{2}{|l|}{6854} \\
\hline Independent reflections & \multicolumn{2}{|c|}{$2473[\mathrm{R}$ (int) $=0.2631]$} \\
\hline Completeness to theta $=23.29^{\circ}$ & \multicolumn{2}{|l|}{$99.5 \%$} \\
\hline Refinement method & \multicolumn{2}{|c|}{ Full-matrix least-squares on $\mathrm{F}^{2}$} \\
\hline Data / restraints / parameters & \multicolumn{2}{|l|}{$2473 / 0$ / 203} \\
\hline Goodness-of-fit on $\mathrm{F}^{2}$ & \multicolumn{2}{|l|}{1.024} \\
\hline Final $R$ indices $[\mathrm{I}>2 \operatorname{sigma}(\mathrm{I})]$ & \multicolumn{2}{|c|}{$\mathrm{R} 1=0.0878, \mathrm{wR} 2=0.2031$} \\
\hline $\mathrm{R}$ indices (all data) & \multicolumn{2}{|c|}{$\mathrm{R} 1=0.0906, \mathrm{wR} 2=0.2070$} \\
\hline Absolute structure parameter & \multicolumn{2}{|l|}{$0.00(3)$} \\
\hline Extinction coefficient & \multicolumn{2}{|l|}{$0.021(3)$} \\
\hline Largest diff. peak and hole & \multicolumn{2}{|c|}{1.886 and -2.216 e. $\AA^{-3}$} \\
\hline
\end{tabular}


Table 2. Atomic coordinates $\left(\times 10^{4}\right)$ and equivalent isotropic displacement parameters $\left(\AA^{2} \times 10^{3}\right)$ for 02015am2.

$\mathrm{U}(\mathrm{eq})$ is defined as one third of the trace of the orthogonalized $\mathrm{U}^{\mathrm{ij}}$ tensor.

\begin{tabular}{|c|c|c|c|c|}
\hline & $x$ & $\mathrm{y}$ & $\mathrm{z}$ & $\mathrm{U}(\mathrm{eq})$ \\
\hline $\operatorname{Br}(1)$ & $1390(2)$ & $3465(1)$ & $8123(1)$ & $38(1)$ \\
\hline $\operatorname{Br}(2)$ & $10818(2)$ & $2670(1)$ & $5866(1)$ & $43(1)$ \\
\hline $\operatorname{Br}(3)$ & $7911(2)$ & 1921(1) & 7011(1) & $43(1)$ \\
\hline $\mathrm{O}(4)$ & $4226(15)$ & $4690(7)$ & $8955(3)$ & $33(2)$ \\
\hline $\mathrm{O}(1)$ & $7270(13)$ & $6259(6)$ & $5707(4)$ & $30(2)$ \\
\hline $\mathrm{O}(3)$ & 6186(19) & $2986(7)$ & $5694(4)$ & $52(3)$ \\
\hline $\mathrm{O}(2)$ & $9753(12)$ & $5380(7)$ & $6174(4)$ & $33(2)$ \\
\hline$C(10)$ & $4873(18)$ & $4746(9)$ & $8315(5)$ & $22(2)$ \\
\hline$C(9)$ & $3690(20)$ & 4192(9) & $7855(5)$ & $29(3)$ \\
\hline$C(8)$ & $4205(18)$ & 4191(9) & $7202(5)$ & $24(2)$ \\
\hline$C(7)$ & $5892(18)$ & $4715(8)$ & $6980(5)$ & $23(2)$ \\
\hline$C(12)$ & $7078(18)$ & $5243(9)$ & 7433(5) & $24(2)$ \\
\hline$C(11)$ & $6507(17)$ & $5260(9)$ & $8104(5)$ & $27(2)$ \\
\hline$C(13)$ & $5230(20)$ & $5340(11)$ & $9432(5)$ & $40(3)$ \\
\hline$C(15)$ & $8790(20)$ & $3108(9)$ & $6485(5)$ & $28(3)$ \\
\hline$C(14)$ & $7020(20)$ & $3539(10)$ & $6086(5)$ & $29(3)$ \\
\hline$C(2)$ & $6368(18)$ & $4665(9)$ & $6238(5)$ & $21(2)$ \\
\hline$C(1)$ & $8017(18)$ & $5464(9)$ & $6051(5)$ & $26(3)$ \\
\hline$C(4)$ & $5058(19)$ & $6211(9)$ & $5644(5)$ & $25(2)$ \\
\hline$C(3)$ & $4696(17)$ & $5021(9)$ & $5785(5)$ & $26(2)$ \\
\hline$C(5)$ & $4160(20)$ & $6940(11)$ & $6127(6)$ & $41(3)$ \\
\hline$C(6)$ & $4650(20)$ & $6524(10)$ & $4938(6)$ & $40(3)$ \\
\hline
\end{tabular}


Table 3. Bond lengths $[\AA]$ and angles $\left[^{\circ}\right]$ for 02015am2.

\begin{tabular}{|c|c|}
\hline $\mathrm{Br}(1)-\mathrm{C}(9)$ & $1.884(13)$ \\
\hline $\operatorname{Br}(2)-C(15)$ & $1.939(12)$ \\
\hline $\mathrm{Br}(3)-\mathrm{C}(15)$ & $1.930(11)$ \\
\hline $\mathrm{O}(4)-\mathrm{C}(10)$ & $1.373(13)$ \\
\hline $\mathrm{O}(4)-\mathrm{C}(13)$ & $1.437(15)$ \\
\hline $\mathrm{O}(1)-\mathrm{C}(1)$ & $1.321(15)$ \\
\hline $\mathrm{O}(1)-\mathrm{C}(4)$ & $1.499(16)$ \\
\hline $\mathrm{O}(3)-\mathrm{C}(14)$ & $1.198(14)$ \\
\hline $\mathrm{O}(2)-\mathrm{C}(1)$ & $1.203(16)$ \\
\hline$C(10)-C(11)$ & $1.348(17)$ \\
\hline$C(10)-C(9)$ & $1.412(17)$ \\
\hline$C(9)-C(8)$ & $1.371(16)$ \\
\hline$C(8)-C(7)$ & $1.391(17)$ \\
\hline$C(7)-C(12)$ & $1.388(16)$ \\
\hline$C(7)-C(2)$ & $1.542(13)$ \\
\hline$C(12)-C(11)$ & $1.417(15)$ \\
\hline$C(15)-C(14)$ & $1.543(18)$ \\
\hline$C(14)-C(2)$ & $1.516(16)$ \\
\hline$C(2)-C(3)$ & $1.524(17)$ \\
\hline$C(2)-C(1)$ & $1.548(15)$ \\
\hline$C(4)-C(5)$ & $1.475(18)$ \\
\hline$C(4)-C(6)$ & $1.513(15)$ \\
\hline$C(4)-C(3)$ & $1.544(17)$ \\
\hline $\mathrm{C}(10)-\mathrm{O}(4)-\mathrm{C}(13)$ & $117.4(11)$ \\
\hline $\mathrm{C}(1)-\mathrm{O}(1)-\mathrm{C}(4)$ & 113.2(9) \\
\hline $\mathrm{C}(11)-\mathrm{C}(10)-\mathrm{O}(4)$ & $125.8(11)$ \\
\hline$C(11)-C(10)-C(9)$ & $119.3(10)$ \\
\hline $\mathrm{O}(4)-\mathrm{C}(10)-\mathrm{C}(9)$ & $114.9(11)$ \\
\hline$C(8)-C(9)-C(10)$ & $119.8(12)$ \\
\hline$C(8)-C(9)-B r(1)$ & $119.2(9)$ \\
\hline$C(10)-C(9)-B r(1)$ & $121.0(9)$ \\
\hline$C(9)-C(8)-C(7)$ & $121.3(11)$ \\
\hline$C(12)-C(7)-C(8)$ & $119.0(9)$ \\
\hline$C(12)-C(7)-C(2)$ & 123.1(10) \\
\hline$C(8)-C(7)-C(2)$ & $117.9(10)$ \\
\hline$C(7)-C(12)-C(11)$ & $119.2(11)$ \\
\hline$C(10)-C(11)-C(12)$ & $121.4(10)$ \\
\hline$C(14)-C(15)-B r(3)$ & $109.0(9)$ \\
\hline$C(14)-C(15)-B r(2)$ & $107.8(7)$ \\
\hline $\mathrm{Br}(3)-\mathrm{C}(15)-\mathrm{Br}(2)$ & $110.8(5)$ \\
\hline $\mathrm{O}(3)-\mathrm{C}(14)-\mathrm{C}(2)$ & $122.9(12)$ \\
\hline $\mathrm{O}(3)-\mathrm{C}(14)-\mathrm{C}(15)$ & $120.5(11)$ \\
\hline$C(2)-C(14)-C(15)$ & $116.5(10)$ \\
\hline
\end{tabular}




$\begin{array}{ll}\mathrm{C}(14)-\mathrm{C}(2)-\mathrm{C}(3) & 111.5(9) \\ \mathrm{C}(14)-\mathrm{C}(2)-\mathrm{C}(7) & 107.3(9) \\ \mathrm{C}(3)-\mathrm{C}(2)-\mathrm{C}(7) & 115.0(10) \\ \mathrm{C}(14)-\mathrm{C}(2)-\mathrm{C}(1) & 110.5(10) \\ \mathrm{C}(3)-\mathrm{C}(2)-\mathrm{C}(1) & 101.1(9) \\ \mathrm{C}(7)-\mathrm{C}(2)-\mathrm{C}(1) & 111.3(9) \\ \mathrm{O}(2)-\mathrm{C}(1)-\mathrm{O}(1) & 123.3(10) \\ \mathrm{O}(2)-\mathrm{C}(1)-\mathrm{C}(2) & 126.3(11) \\ \mathrm{O}(1)-\mathrm{C}(1)-\mathrm{C}(2) & 110.4(10) \\ \mathrm{C}(5)-\mathrm{C}(4)-\mathrm{O}(1) & 109.2(10) \\ \mathrm{C}(5)-\mathrm{C}(4)-\mathrm{C}(6) & 113.1(10) \\ \mathrm{O}(1)-\mathrm{C}(4)-\mathrm{C}(6) & 104.6(10) \\ \mathrm{C}(5)-\mathrm{C}(4)-\mathrm{C}(3) & 114.5(10) \\ \mathrm{O}(1)-\mathrm{C}(4)-\mathrm{C}(3) & 100.5(10) \\ \mathrm{C}(6)-\mathrm{C}(4)-\mathrm{C}(3) & 113.5(9) \\ \mathrm{C}(2)-\mathrm{C}(3)-\mathrm{C}(4) & 106.3(10) \\ \end{array}$


Table 4. Anisotropic displacement parameters $\left(\AA^{2} \times 10^{3}\right)$ for $02015 \mathrm{am} 2$.

The anisotropic displacement factor exponent takes the form: $-2_{-}^{2}\left[h^{2} a^{* 2} U^{11}+\ldots+2 h k a^{*} b^{*} U^{12}\right]$

\begin{tabular}{|c|c|c|c|c|c|c|}
\hline & $\mathrm{U}^{11}$ & $\mathrm{U}^{22}$ & $\mathrm{U}^{33}$ & $\mathrm{U}^{23}$ & $\mathrm{U}^{13}$ & $\mathrm{U}^{12}$ \\
\hline $\operatorname{Br}(1)$ & $33(1)$ & $61(1)$ & 19(1) & $6(1)$ & $3(1)$ & $-13(1)$ \\
\hline $\operatorname{Br}(2)$ & $46(1)$ & $47(1)$ & $34(1)$ & $-2(1)$ & $15(1)$ & $9(1)$ \\
\hline $\operatorname{Br}(3)$ & $49(1)$ & $48(1)$ & $32(1)$ & $15(1)$ & $-4(1)$ & $-14(1)$ \\
\hline $\mathrm{O}(4)$ & $43(5)$ & $53(5)$ & $3(3)$ & $-2(3)$ & $6(4)$ & $4(4)$ \\
\hline $\mathrm{O}(1)$ & $31(5)$ & $34(4)$ & $24(4)$ & $7(3)$ & $7(3)$ & $-6(4)$ \\
\hline $\mathrm{O}(3)$ & $75(8)$ & $36(4)$ & $45(5)$ & $-23(4)$ & $-42(6)$ & $4(5)$ \\
\hline $\mathrm{O}(2)$ & $19(4)$ & $50(5)$ & $29(4)$ & $4(4)$ & $0(3)$ & $-1(4)$ \\
\hline$C(10)$ & $24(6)$ & $27(5)$ & $15(5)$ & $0(4)$ & $1(4)$ & $6(5)$ \\
\hline$C(9)$ & $34(6)$ & $28(5)$ & $24(5)$ & $-5(5)$ & $-1(5)$ & $1(5)$ \\
\hline$C(8)$ & $27(6)$ & $31(5)$ & $12(5)$ & $-1(4)$ & $1(5)$ & $-4(5)$ \\
\hline$C(7)$ & $27(6)$ & $34(5)$ & $8(5)$ & $7(5)$ & $3(5)$ & $-4(5)$ \\
\hline$C(12)$ & $24(6)$ & $39(6)$ & $7(4)$ & $5(4)$ & $-1(5)$ & $1(5)$ \\
\hline$C(11)$ & $29(6)$ & $31(6)$ & $21(5)$ & $-12(5)$ & $-3(5)$ & $-3(5)$ \\
\hline$C(13)$ & $49(9)$ & $52(7)$ & $17(6)$ & $-16(6)$ & $-7(5)$ & $-2(7)$ \\
\hline$C(15)$ & $44(8)$ & $23(5)$ & 19(5) & $3(5)$ & $-4(5)$ & $2(6)$ \\
\hline$C(14)$ & $37(7)$ & $40(6)$ & $10(5)$ & 1(5) & $0(5)$ & $0(6)$ \\
\hline$C(2)$ & $26(6)$ & $31(5)$ & $6(4)$ & $-7(4)$ & $4(4)$ & $-8(5)$ \\
\hline$C(1)$ & $33(7)$ & $36(6)$ & $9(5)$ & $-11(5)$ & $8(5)$ & $-14(6)$ \\
\hline$C(4)$ & $28(6)$ & $32(6)$ & $15(5)$ & $6(5)$ & $-6(4)$ & $-3(5)$ \\
\hline$C(3)$ & $23(6)$ & $40(6)$ & $14(5)$ & $-7(5)$ & $-1(5)$ & $4(5)$ \\
\hline$C(5)$ & $30(7)$ & $50(7)$ & $43(7)$ & $-11(7)$ & $-3(6)$ & $3(6)$ \\
\hline$C(6)$ & $53(9)$ & $39(7)$ & $28(6)$ & $15(5)$ & $-8(6)$ & $9(7)$ \\
\hline
\end{tabular}


Table 5. Hydrogen coordinates ( x 104) and isotropic displacement parameters $\left(\AA^{2} \times 10^{3}\right)$ for 02015am2.

\begin{tabular}{|c|c|c|c|c|}
\hline & $\mathrm{x}$ & $\mathrm{y}$ & z & $\mathrm{U}(\mathrm{eq})$ \\
\hline $\mathrm{H}(8)$ & 3393 & 3825 & 6895 & 28 \\
\hline $\mathrm{H}(12)$ & 8257 & 5589 & 7294 & 28 \\
\hline $\mathrm{H}(11)$ & 7292 & 5641 & 8412 & 32 \\
\hline $\mathrm{H}(13 \mathrm{~A})$ & 6663 & 5266 & 9377 & 59 \\
\hline $\mathrm{H}(13 \mathrm{~B})$ & 4852 & 5112 & 9877 & 59 \\
\hline $\mathrm{H}(13 \mathrm{C})$ & 4848 & 6084 & 9369 & 59 \\
\hline $\mathrm{H}(15)$ & 9320 & 3678 & 6779 & 34 \\
\hline $\mathrm{H}(3 \mathrm{~A})$ & 3395 & 4918 & 6001 & 31 \\
\hline $\mathrm{H}(3 \mathrm{~B})$ & 4714 & 4609 & 5370 & 31 \\
\hline $\mathrm{H}(5 \mathrm{~A})$ & 4529 & 6719 & 6574 & 61 \\
\hline $\mathrm{H}(5 \mathrm{~B})$ & 2712 & 6920 & 6083 & 61 \\
\hline $\mathrm{H}(5 \mathrm{C})$ & 4633 & 7663 & 6047 & 61 \\
\hline $\mathrm{H}(6 \mathrm{~A})$ & 3341 & 6254 & 4806 & 60 \\
\hline $\mathrm{H}(6 \mathrm{~B})$ & 5665 & 6220 & 4651 & 60 \\
\hline $\mathrm{H}(6 \mathrm{C})$ & 4662 & 7299 & 4899 & 60 \\
\hline
\end{tabular}


Table 6. Torsion angles $\left[{ }^{\circ}\right]$ for 02015am2.

\begin{tabular}{|c|c|}
\hline $\mathrm{C}(13)-\mathrm{O}(4)-\mathrm{C}(10)-\mathrm{C}(11)$ & $8.2(17)$ \\
\hline $\mathrm{C}(13)-\mathrm{O}(4)-\mathrm{C}(10)-\mathrm{C}(9)$ & $-172.7(11)$ \\
\hline$C(11)-C(10)-C(9)-C(8)$ & $-0.6(17)$ \\
\hline $\mathrm{O}(4)-\mathrm{C}(10)-\mathrm{C}(9)-\mathrm{C}(8)$ & $-179.7(11)$ \\
\hline$C(11)-C(10)-C(9)-B r(1)$ & $-179.8(9)$ \\
\hline $\mathrm{O}(4)-\mathrm{C}(10)-\mathrm{C}(9)-\mathrm{Br}(1)$ & $1.0(14)$ \\
\hline$C(10)-C(9)-C(8)-C(7)$ & $0.9(17)$ \\
\hline $\mathrm{Br}(1)-\mathrm{C}(9)-\mathrm{C}(8)-\mathrm{C}(7)$ & $-179.9(9)$ \\
\hline$C(9)-C(8)-C(7)-C(12)$ & $0.4(17)$ \\
\hline$C(9)-C(8)-C(7)-C(2)$ & $179.5(11)$ \\
\hline$C(8)-C(7)-C(12)-C(11)$ & $-1.8(16)$ \\
\hline$C(2)-C(7)-C(12)-C(11)$ & 179.1(11) \\
\hline $\mathrm{O}(4)-\mathrm{C}(10)-\mathrm{C}(11)-\mathrm{C}(12)$ & 178.2(11) \\
\hline$C(9)-C(10)-C(11)-C(12)$ & $-0.9(17)$ \\
\hline$C(7)-C(12)-C(11)-C(10)$ & $2.1(17)$ \\
\hline $\mathrm{Br}(3)-\mathrm{C}(15)-\mathrm{C}(14)-\mathrm{O}(3)$ & $-61.2(13)$ \\
\hline $\operatorname{Br}(2)-C(15)-C(14)-O(3)$ & $59.2(14)$ \\
\hline $\mathrm{Br}(3)-\mathrm{C}(15)-\mathrm{C}(14)-\mathrm{C}(2)$ & $116.5(9)$ \\
\hline $\operatorname{Br}(2)-C(15)-C(14)-C(2)$ & $-123.2(9)$ \\
\hline $\mathrm{O}(3)-\mathrm{C}(14)-\mathrm{C}(2)-\mathrm{C}(3)$ & $-5.8(16)$ \\
\hline$C(15)-C(14)-C(2)-C(3)$ & $176.6(9)$ \\
\hline $\mathrm{O}(3)-\mathrm{C}(14)-\mathrm{C}(2)-\mathrm{C}(7)$ & $121.1(13)$ \\
\hline$C(15)-C(14)-C(2)-C(7)$ & $-56.5(13)$ \\
\hline $\mathrm{O}(3)-\mathrm{C}(14)-\mathrm{C}(2)-\mathrm{C}(1)$ & $-117.4(13)$ \\
\hline$C(15)-C(14)-C(2)-C(1)$ & $65.0(11)$ \\
\hline$C(12)-C(7)-C(2)-C(14)$ & $108.5(12)$ \\
\hline $\mathrm{C}(8)-\mathrm{C}(7)-\mathrm{C}(2)-\mathrm{C}(14)$ & $-70.6(13)$ \\
\hline$C(12)-C(7)-C(2)-C(3)$ & $-126.8(12)$ \\
\hline$C(8)-C(7)-C(2)-C(3)$ & $54.2(14)$ \\
\hline$C(12)-C(7)-C(2)-C(1)$ & $-12.5(15)$ \\
\hline$C(8)-C(7)-C(2)-C(1)$ & $168.4(10)$ \\
\hline $\mathrm{C}(4)-\mathrm{O}(1)-\mathrm{C}(1)-\mathrm{O}(2)$ & $-177.5(10)$ \\
\hline $\mathrm{C}(4)-\mathrm{O}(1)-\mathrm{C}(1)-\mathrm{C}(2)$ & $4.7(12)$ \\
\hline $\mathrm{C}(14)-\mathrm{C}(2)-\mathrm{C}(1)-\mathrm{O}(2)$ & $-45.6(14)$ \\
\hline $\mathrm{C}(3)-\mathrm{C}(2)-\mathrm{C}(1)-\mathrm{O}(2)$ & $-163.8(11)$ \\
\hline $\mathrm{C}(7)-\mathrm{C}(2)-\mathrm{C}(1)-\mathrm{O}(2)$ & $73.6(14)$ \\
\hline $\mathrm{C}(14)-\mathrm{C}(2)-\mathrm{C}(1)-\mathrm{O}(1)$ & 132.2(9) \\
\hline $\mathrm{C}(3)-\mathrm{C}(2)-\mathrm{C}(1)-\mathrm{O}(1)$ & $14.0(11)$ \\
\hline $\mathrm{C}(7)-\mathrm{C}(2)-\mathrm{C}(1)-\mathrm{O}(1)$ & $-108.7(10)$ \\
\hline $\mathrm{C}(1)-\mathrm{O}(1)-\mathrm{C}(4)-\mathrm{C}(5)$ & $99.8(11)$ \\
\hline $\mathrm{C}(1)-\mathrm{O}(1)-\mathrm{C}(4)-\mathrm{C}(6)$ & $-138.8(9)$ \\
\hline $\mathrm{C}(1)-\mathrm{O}(1)-\mathrm{C}(4)-\mathrm{C}(3)$ & $-20.9(11)$ \\
\hline$C(14)-C(2)-C(3)-C(4)$ & $-143.8(9)$ \\
\hline$C(7)-C(2)-C(3)-C(4)$ & $93.6(11)$ \\
\hline
\end{tabular}


$\mathrm{C}(1)-\mathrm{C}(2)-\mathrm{C}(3)-\mathrm{C}(4)$

$\mathrm{C}(5)-\mathrm{C}(4)-\mathrm{C}(3)-\mathrm{C}(2)$

$\mathrm{O}(1)-\mathrm{C}(4)-\mathrm{C}(3)-\mathrm{C}(2)$

$\mathrm{C}(6)-\mathrm{C}(4)-\mathrm{C}(3)-\mathrm{C}(2)$
$-26.4(10)$

$-88.1(13)$

28.8(10)

139.9(11) 